\title{
On the discounted aggregate claim costs until ruin in dependent Sparre Andersen risk processes
}

\author{
Eric C.K. Cheung* and Jae-Kyung Woo*†
}

February 24, 2014

\begin{abstract}
In this paper, a dependent Sparre Andersen risk process in which the joint density of the interclaim time and the resulting claim severity satisfies the factorization as in Willmot and Woo (2012) is considered. We study a generalization of the Gerber-Shiu function (i) whose penalty function further depends on the surplus level immediately after the second last claim before ruin (Cheung et al. (2010a)); and (ii) which involves the moments of the discounted aggregate claim costs until ruin. The generalized discounted density with a moment-based component proposed in Cheung (2013) plays a key role in deriving recursive defective renewal equations. We pay special attention to the case where the marginal distribution of the interclaim times is Coxian, and the required components in the recursion are obtained. A reverse type of dependency structure where the claim severities follow a combination of exponentials is also briefly discussed, and this leads to a nice explicit expression for the expected discounted aggregate claims until ruin. Our results are applied to generate some numerical examples involving (i) the covariance of the time of ruin and the discounted aggregate claims until ruin; and (ii) the expectation, variance and third central moment of the discounted aggregate claims until ruin.
\end{abstract}

Keywords: Dependent Sparre Andersen risk model; Discounted aggregate claims until ruin; Discounted densities; Coxian distribution; Higher moments; Covariance.

\section{Introduction}

Let us consider the surplus process $\{U(t)\}_{t \geq 0}$ of an insurance company, where $U(t)$ denotes the insurer's surplus level at time $t$ given by

$$
U(t)=u+c t-\sum_{i=1}^{N(t)} Y_{i}, \quad t \geq 0 .
$$

Here $u=U(0) \geq 0$ is the initial surplus and $c>0$ is the premium collected per unit time. We assume that the claim number process $\{N(t)\}_{t \geq 0}$ is a renewal process corresponding to the sequence of independent and identically distributed (i.i.d.) positive continuous interclaim times $\left\{V_{i}\right\}_{i=1}^{\infty}$ (with generic random variable $V$ ). In particular, $V_{1}$ is the time of the first claim and $V_{i}$ is the time between the $(i-1)$-th

\footnotetext{
${ }^{*}$ Department of Statistics and Actuarial Science, University of Hong Kong, Pokfulam Road, Hong Kong.

${ }^{\dagger}$ Corresponding author. Email address: jkwoo@hku.hk
} 
and the $i$-th claims for $i=2,3, \ldots$ Moreover, $Y_{i}$ denotes the size of the $i$-th claim for $i=1,2, \ldots$, and $\left\{Y_{i}\right\}_{i=1}^{\infty}$ is assumed to form an i.i.d. sequence of positive continuous random variables having the same distribution as $Y$. Defining the time of ruin to be $\tau=\inf \{t \geq 0: U(t)<0\}$ (with $\tau=\infty$ if $U(t) \geq 0$ for all $t \geq 0$ ), it is known that the positive security loading condition $c E[V]>E[Y]$ ensures that the ruin probability $\psi(u)=\operatorname{Pr}\{\tau<\infty \mid U(0)=u\}$ is strictly less than 1 (e.g. Prabhu (1998, Part I)).

In the traditional Sparre Andersen or renewal risk model (Sparre Andersen (1957)), the i.i.d. sequences $\left\{V_{i}\right\}_{i=1}^{\infty}$ and $\left\{Y_{i}\right\}_{i=1}^{\infty}$ are typically assumed to be mutually independent. Recently, there has been increased research interests in the more general class of dependent Sparre Andersen risk processes which only assumes that the pairs $\left\{\left(V_{i}, Y_{i}\right)\right\}_{i=1}^{\infty}$ are i.i.d., i.e. the $i$-th claim severity $Y_{i}$ is allowed to possibly depend on the elapsed time $V_{i}$ since the last claim. Under such a relaxation, the surplus process $\{U(t)\}_{t \geq 0}$ still retains a random walk structure at claim instants since the increments $\left\{c V_{i}-Y_{i}\right\}_{i=1}^{\infty}$ form an i.i.d. sequence. Various general results in dependent Sparre Andersen risk models are available in the literature. For example, asymptotic expressions concerning (finite-time) ruin probabilities for light-tailed claims were given by Albrecher and Teugels (2006); whereas Cheung et al. (2010b) studied the structural properties of the Gerber-Shiu function (Gerber and Shiu (1998)) with a generalized penalty. Some of the latter results were further extended to the delayed (Woo (2010)) and discrete (Woo (2012)) processes as well as models with surplus-dependent premium (Cheung (2011)). For the study of ruin-related quantities in dependent Sparre Andersen models under specific distributional assumptions on the interclaim time $V$ and/or the claim severity $Y$, we refer interested readers to e.g. Boudreault et al. (2006), Cossette et al. (2008, 2010), Ambagaspitiya (2009), Badescu et al. (2009), Chadjiconstantinidis and Vrontos (2012), Willmot and Woo (2012), Zhang et al. (2012), and Landriault et al. (2013). In this paper, we are mostly concerned with the dependency structure proposed by Willmot and Woo (2012), who assumed that the joint density of the generic pair $(V, Y)$ at $(t, y)$ admits the factorization form

$$
p(t, y)=\sum_{i=1}^{r} \sum_{j=1}^{m_{i}} k_{i j}(t) b_{i j}(y), \quad t, y>0 .
$$

The above double indexing, though not strictly necessary, will facilitate presentation in Section 3 . Note that $k_{i j}(\cdot)$ and $b_{i j}(\cdot)$ are not necessarily probability density functions, as long as $p(\cdot, \cdot)$ is a valid joint probability density. The joint Laplace transform of $(V, Y)$ is defined by

$$
\widetilde{p}\left(s_{1}, s_{2}\right)=\sum_{i=1}^{r} \sum_{j=1}^{m_{i}} \widetilde{k}_{i j}\left(s_{1}\right) \widetilde{b}_{i j}\left(s_{2}\right),
$$

where $\widetilde{k}_{i j}(s)=\int_{0}^{\infty} e^{-s t} k_{i j}(t) d t$ and $\widetilde{b}_{i j}(s)=\int_{0}^{\infty} e^{-s y} b_{i j}(y) d y$. For later use, we also write $p(t, y)=$ $p_{t}(y) k(t)$, where $k(\cdot)$ is the density of $V$ and $p_{t}(\cdot)$ is the conditional density of $Y$ given $V=t$, i.e. $p_{t}(y)=P_{t}^{\prime}(y)$ with $P_{t}(y)=\operatorname{Pr}(Y \leq y \mid V=t)$. Also define the Laplace transforms $\widetilde{k}(s)=\int_{0}^{\infty} e^{-s t} k(t) d t$ and $\widetilde{p}_{t}(s)=\int_{0}^{\infty} e^{-s y} p_{t}(y) d y$.

It is well known that the Gerber-Shiu expected discounted penalty function proposed by Gerber and Shiu (1998) involves three random variables, namely the time of ruin $\tau$, the surplus immediately prior to ruin $U\left(\tau^{-}\right)$, and the deficit at ruin $|U(\tau)|$. While the time $\tau$ appears through a discount factor, the variables $U\left(\tau^{-}\right)$and $|U(\tau)|$ are incorporated into the so-called penalty function. Our goal is to analyze a Gerber-Shiu type function which additionally depends on two variables. First, define $R_{i}=u+\sum_{j=1}^{i}\left(c V_{j}-Y_{j}\right)$ to be the surplus level immediately after the $i$-th claim for $i=1,2, \ldots$ with $R_{0}=u$. In the spirit of Cheung et al. (2010a), we shall further incorporate the surplus immediately 
after the second last claim before ruin, namely $R_{N(\tau)-1}$, into the penalty function. As illustrated by e.g. Cheung et al. (2011), the variable $R_{N(\tau)-1}$ can be used to study the last interclaim time before ruin due to the relationship $V_{N(\tau)}=\left(U\left(\tau^{-}\right)-R_{N(\tau)-1}\right) / c$. Next, denoting the occurrence time of the $i$-th claim by $T_{i}=\sum_{j=1}^{i} V_{j}$ for $i=1,2, \ldots$ (with the convention $T_{0}=0$ ), we are also interested in the discounted aggregate claim costs until ruin $Z_{\delta}(\tau)$, where

$$
Z_{\delta}(t)=\sum_{i=1}^{N(t)} e^{-\delta T_{i}} f\left(Y_{i}\right), \quad t \geq 0 .
$$

Here $f(\cdot)$ can be interpreted as the 'cost function' that determines the cost of each claim. Under the classical compound Poisson and the phase-type renewal models, Cai et al. (2009, Section 6) and Feng (2009a, Section 4.2, and 2009b, Section 5.2) respectively studied the expectation of $Z_{\delta}(\tau)$. Higher moments of $Z_{\delta}(\tau)$ were also considered by Cheung and Feng (2013, Section 2.1) when claims occur according to a Markovian arrival process (e.g. Asmussen (2003, Chapter XI.1)) and by Cheung (2013) for a traditional Sparre Andersen model. (Note that the class of risk processes with Markovian claim arrivals and the class of dependent Sparre Andersen risk processes considered in this paper are not special cases of each other.) With the above descriptions, we shall analyze the Gerber-Shiu type function defined by, for $n=0,1, \ldots$,

$$
\phi_{\delta_{1}, \delta_{2}, n}(u)=\phi_{\delta_{12}, n}(u)=E\left[e^{-\delta_{1} \tau} Z_{\delta_{2}}^{n}(\tau) w\left(U\left(\tau^{-}\right),|U(\tau)|, R_{N(\tau)-1}\right) I\{\tau<\infty\} \mid U(0)=u\right], \quad u \geq 0,
$$

where $w$ is the three-variable penalty function satisfying mild integrable condition; $I\{A\}$ is the usual indicator function of the event $A ; \delta_{1} \geq 0$ can either be viewed as a force of interest or a Laplace transform argument with respect to $\tau ; \delta_{2} \geq 0$ is the force of interest used for discounting the claim costs; and $n$ is a non-negative integer representing the order of moment of $Z_{\delta_{2}}(\tau)$. The special case of (1.3) in which $\delta_{1}=n_{1} \delta_{2}$ for some non-negative integer $n_{1}$ and $w(x, y, v)$ only depends on $y$ was first proposed by Cheung (2013, Equation (1.1)), who also discussed various choices of $f(\cdot)$ (e.g. in relation to the number of claims until ruin $N(\tau)$ ). The present use of different $\delta$ 's in $e^{-\delta_{1} \tau}$ and $Z_{\delta_{2}}(\tau)$ will facilitate the study of the covariance of $\tau$ and $Z_{\delta_{2}}(\tau)$ (see Section 5). Moreover, the calculations of the moments of $Z_{\delta_{2}}(\tau)$ allow the possibility to estimate the distribution of $Z_{\delta_{2}}(\tau)$ (see e.g. Lindsay et al. (2000)). For notational convenience, we shall write $\phi_{\delta_{12}, n}(\cdot)$ instead of $\phi_{\delta_{1}, \delta_{2}, n}(\cdot)$ whenever this does not cause any confusion. Similar abbreviations will be adopted for other related functions as well. For simplicity, the variables $Z_{\delta_{2}}(\tau), U\left(\tau^{-}\right),|U(\tau)|$ and $R_{N(\tau)-1}$ will sometimes be denoted by $Z_{\delta_{2}}, U^{-},|U|$ and $R$ respectively.

The rest of this paper is organized as follows. In Section 2, we begin by introducing the moment-based discounted densities associated to the variables $\left(\tau, Z_{\delta_{2}}, U^{-},|U|, R\right)$. These will be used to derive recursive defective renewal equations satisfied by the Gerber-Shiu function $\phi_{\delta_{12}, n}(\cdot)$ for $n=0,1, \ldots$ under a general setting of risk process. In addition, recursive formulas for the generalized discounted densities will be given. The components required in the above recursions are identified for Coxian interclaim times in Section 3. Section 4 revisits a reverse dependency structure in which the claims follow a combination of exponentials, and an explicit expression for the expected discounted aggregate claims until ruin is derived. Our results are then applied in Section 5 to find the covariance of $\tau$ and $\sum_{i=1}^{N(\tau)} e^{-\delta_{2} T_{i}} Y_{i}$ as well as the first three moments of $\sum_{i=1}^{N(\tau)} e^{-\delta_{2} T_{i}} Y_{i}$ through some numerical examples.

\section{Moment-based discounted densities and defective renewal equations}

In order to discuss the moment-based discounted density of the triplet $\left(U^{-},|U|, R\right)$, we begin by introducing the joint distribution of the quintuple $\left(\tau, Z_{\delta_{2}}, U^{-},|U|, R\right)$. Depending on whether ruin occurs 
upon the first claim or not, the joint density has a different form. Suppose $U(0)=u \geq 0$. If the first claim causes ruin (i.e. $N(\tau)=1$ ), then $R=u$, and the time of ruin $\tau$ and the discounted claim cost $Z_{\delta_{2}}$ are characterized by the surplus prior to ruin $U^{-}$and the deficit at ruin $|U|$. More precisely, it is easy to see that $\tau=\left(U^{-}-u\right) / c$ and $Z_{\delta_{2}}=e^{-\delta_{2}\left(U^{-}-u\right) / c} f\left(U^{-}+|U|\right)$. Hence, the joint distribution of $\left(\tau, Z_{\delta_{2}}, U^{-},|U|, R\right)$ in this situation is essentially characterized by the joint density of $\left(U^{-},|U|\right)$ at $(x, y)$, namely (see e.g. Cheung et al. $(2010 \mathrm{a}, \mathrm{b})$ )

$$
g_{1}(x, y \mid u)=\frac{1}{c} p\left(\frac{x-u}{c}, x+y\right), \quad x>u ; y>0 .
$$

On the other hand, if ruin does not occur upon the first claim but on subsequent ones, then no simple relationship holds among these variables, and the joint density of $\left(\tau, Z_{\delta_{2}}, U^{-},|U|, R\right)$ at $(t, z, x, y, v)$ is denoted by $g_{2, \delta_{2}}(t, z, x, y, v \mid u)$ for $0 \leq v<x ; y>0 ; t>\max (x-\min (u, v), 0) / c$ and $z>e^{-\delta_{2} t} f(x+y)$.

With the above definitions, the moment-based discounted densities associated with $g_{1}$ and $g_{2, \delta_{2}}$ are given by

$$
h_{\delta_{1}, \delta_{2}, n}^{(1)}(x, y \mid u)=e^{-\left(\delta_{1}+n \delta_{2}\right)\left(\frac{x-u}{c}\right)} f^{n}(x+y) g_{1}(x, y \mid u), \quad x>u ; y>0
$$

and

$$
h_{\delta_{1}, \delta_{2}, n}^{(2)}(x, y, v \mid u)=\int_{\max (x-\min (u, v), 0) / c}^{\infty} \int_{e^{-\delta_{2} t} f(x+y)}^{\infty} e^{-\delta_{1} t} z^{n} g_{2, \delta_{2}}(t, z, x, y, v \mid u) d z d t, \quad 0 \leq v<x ; y>0,
$$

respectively. Then the Gerber-Shiu type function $\phi_{\delta_{12}, n}(u)$ admits the representation.

$$
\begin{array}{r}
\phi_{\delta_{12}, n}(u)=\int_{0}^{\infty} \int_{u}^{\infty} w(x, y, u) h_{\delta_{1}, \delta_{2}, n}^{(1)}(x, y \mid u) d x d y+\int_{0}^{\infty} \int_{0}^{\infty} \int_{0}^{x} w(x, y, v) h_{\delta_{1}, \delta_{2}, n}^{(2)}(x, y, v \mid u) d v d x d y \\
u \geq 0 .
\end{array}
$$

For later use, we also define the moment-based discounted joint density of the surplus prior to ruin and the deficit at ruin, namely

$$
\bar{h}_{\delta_{1}, \delta_{2}, n}(x, y \mid u)=h_{\delta_{1}, \delta_{2}, n}^{(1)}(x, y \mid u)+\int_{0}^{x} h_{\delta_{1}, \delta_{2}, n}^{(2)}(x, y, v \mid u) d v, \quad x, y>0,
$$

with $h_{\delta_{1}, \delta_{2}, n}^{(1)}(x, y \mid u)$ understood to be 0 when $x \leq u$. Then, the moment-based discounted marginal density of the deficit is

$$
h_{\delta_{1}, \delta_{2}, n}(y \mid u)=\int_{0}^{\infty} \bar{h}_{\delta_{1}, \delta_{2}, n}(x, y \mid u) d x=\int_{0}^{\infty}\left\{h_{\delta_{1}, \delta_{2}, n}^{(1)}(x, y \mid u)+\int_{0}^{x} h_{\delta_{1}, \delta_{2}, n}^{(2)}(x, y, v \mid u) d v\right\} d x, \quad y>0 .
$$

In addition, the argument $\delta_{1}+n \delta_{2}$ will appear in many places in our analysis. To ease presentation, we define the quantity $\delta_{n}^{*}=\delta_{1}+n \delta_{2}$ for $n=0,1, \ldots$ Utilizing the moment-based discounted densities (2.2), (2.3) and (2.6) leads to the following Proposition.

Proposition 1 For $n=0,1, \ldots$, the Gerber-Shiu function $\phi_{\delta_{12}, n}(\cdot)$ defined in (1.3) satisfies the defective renewal equation

$$
\phi_{\delta_{12}, n}(u)=\alpha_{\delta_{n}^{*}} \int_{0}^{u} \phi_{\delta_{12}, n}(u-y) l_{\delta_{n}^{*}}(y) d y+\nu_{\delta_{12}, n}(u), \quad u \geq 0
$$

where

$$
\alpha_{\delta_{n}^{*}}=\int_{0}^{\infty} h_{\delta_{n}^{*}, \delta_{2}, 0}(y \mid 0) d y=E\left[e^{-\delta_{n}^{*} \tau} I\{\tau<\infty\} \mid U(0)=0\right]<1
$$




$$
l_{\delta_{n}^{*}}(y)=\frac{1}{\alpha_{\delta_{n}^{*}}} h_{\delta_{n}^{*}, \delta_{2}, 0}(y \mid 0), \quad y>0
$$

is a proper density, and

$$
\begin{aligned}
& \nu_{\delta_{12}, n}(u)=\sum_{j=0}^{n-1}\left(\begin{array}{l}
n \\
j
\end{array}\right) \int_{0}^{u} h_{\delta_{j}^{*}, \delta_{2}, n-j}(u-s \mid 0) \phi_{\delta_{12}, j}(s) d s \\
&+\int_{0}^{\infty} \int_{u}^{\infty}\left\{w(x, y, u) h_{\delta_{1}, \delta_{2}, n}^{(1)}(x-u, y+u \mid 0)+\int_{u}^{x} w(x, y, v) h_{\delta_{1}, \delta_{2}, n}^{(2)}(x-u, y+u, v-u \mid 0) d v\right\} d x d y \\
& u \geq 0
\end{aligned}
$$

Proof: Arguments analogous to Cheung (2013, Section 2.2) can be applied here. Given the initial surplus level $U(0)=u$, we consider whether ruin occurs at the time of the first drop of the surplus process below $u$. In the case where ruin occurs upon the first drop, two cases need to be further distinguished depending on whether it is caused by the first claim or by subsequent claims. Applying the moment-based discounted densities (2.2), (2.3) and (2.6) with zero initial surplus, we arrive at the integral equation

$$
\begin{aligned}
\phi_{\delta_{12}, n}(u) & =\sum_{j=0}^{n}\left(\begin{array}{l}
n \\
j
\end{array}\right) \int_{0}^{u} h_{\delta_{j}^{*}, \delta_{2}, n-j}(y \mid 0) \phi_{\delta_{12}, j}(u-y) d y \\
& +\int_{u}^{\infty} \int_{0}^{\infty}\left\{w(x+u, y-u, u) h_{\delta_{1}, \delta_{2}, n}^{(1)}(x, y \mid 0)+\int_{0}^{x} w(x+u, y-u, v+u) h_{\delta_{1}, \delta_{2}, n}^{(2)}(x, y, v \mid 0) d v\right\} d x d y .
\end{aligned}
$$

Separating the term $j=n$ in the summation together with a change of variables yields the renewal equation (2.7) with the components defined by (2.8)-(2.10). We remark that the second equality in (2.8) follows from inspection of (2.4) and (2.6) (with $w \equiv 1 ; u=0 ; n$ replaced by 0 ; and $\delta_{1}$ replaced by $\delta_{n}^{*}$ ). Moreover, the inequality $\alpha_{\delta_{n}^{*}}<1$ holds when either $\delta_{n}^{*}>0$ or the loading condition $c E[V]>E[Y]$ holds. Hence, one asserts that $(2.7)$ is a defective renewal equation satisfied by $\phi_{\delta_{12}, n}(\cdot)$.

It is instructive to note that the defective renewal equation (2.7) is recursive in the order of moment $n$ because the term $\nu_{\delta_{12}, n}(\cdot)$ depends on $\phi_{\delta_{12}, j}(\cdot)$ 's for $j=0,1, \ldots, n-1$. In addition, the deficit densities $h_{\delta_{j}^{*}, \delta_{2}, n-j}(\cdot \mid 0)$ 's for $j=1,2, \ldots, n$ appearing in (2.8)-(2.10) are also related to $\phi_{\delta_{j}^{*}, \delta_{2}, n-j}(0)$ 's. Nonetheless, assuming all these lower order attributes have been obtained, the only unknown part in the defective renewal equation $(2.7)$ is $h_{\delta_{1}, \delta_{2}, n}^{(2)}(x, y, v \mid 0)$. (Note that $h_{\delta_{1}, \delta_{2}, n}^{(1)}(x, y \mid 0)$ is explicitly known from $(2.1)$ and (2.2), and $h_{\delta_{0}^{*}, \delta_{2}, n}(y \mid 0)=h_{\delta_{1}, \delta_{2}, n}(y \mid 0)$ depends on $h_{\delta_{1}, \delta_{2}, n}^{(1)}(x, y \mid 0)$ and $h_{\delta_{1}, \delta_{2}, n}^{(2)}(x, y, v \mid 0)$ via (2.6).) Hence, the implication of Proposition 1 is that it is sufficient to determine $h_{\delta_{1}, \delta_{2}, n}^{(2)}(x, y, v \mid 0)$, which is usually done when specific distributional assumption on the interclaim time is made (see Section 4) via Lemma 1. Then, the solution of the defective renewal equation (2.7) is given by (e.g. Resnick (1992, Section $3.5)$ )

$$
\phi_{\delta_{12}, n}(u)=\nu_{\delta_{12}, n}(u)+\frac{1}{1-\alpha_{\delta_{n}^{*}}} \int_{0}^{u} a_{\delta_{n}^{*}}(u-t) \nu_{\delta_{12}, n}(t) d t, \quad u \geq 0
$$

where

$$
a_{\delta_{n}^{*}}(y)=\sum_{k=1}^{\infty}\left(1-\alpha_{\delta_{n}^{*}}\right) \alpha_{\delta_{n}^{*}}^{k} l_{\delta_{n}^{*}}^{* k}(y), \quad y>0
$$

is a compound geometric density. Here $l_{\delta_{n}^{*}}^{* k}(\cdot)$ represents the $k$-fold convolution density of $l_{\delta_{n}^{*}}(\cdot)$ with itself for $k=1,2, \ldots$ 
Next, suppose we have determined $h_{\delta_{1}, \delta_{2}, n}^{(2)}(x, y, v \mid 0)$ (and hence $h_{\delta_{1}, \delta_{2}, n}(y \mid 0)$ ). We shall illustrate that the solution (2.12) can also be used to derive recursive formulas for the moment-based discounted density $h_{\delta_{1}, \delta_{2}, n}^{(2)}(x, y, v \mid u)$ for initial surplus $u \geq 0$, which is sufficient to characterize $\phi_{\delta_{12}, n}(u)$ via (2.4). For now, we focus on the first term on the right-hand side of (2.10). Substitution of (2.4) followed by a change of order of integrals yields

$$
\sum_{j=0}^{n-1}\left(\begin{array}{l}
n \\
j
\end{array}\right) \int_{0}^{u} h_{\delta_{j}^{*}, \delta_{2}, n-j}(u-s \mid 0) \phi_{\delta_{12}, j}(s) d s=\int_{0}^{\infty} \int_{0}^{\infty} \int_{0}^{x} w(x, y, v) \chi_{\delta_{12}, n}(x, y, v \mid u) d v d x d y
$$

where

$$
\begin{gathered}
\chi_{\delta_{12}, n}(x, y, v \mid u)=\sum_{j=0}^{n-1}\left(\begin{array}{c}
n \\
j
\end{array}\right)\left\{h_{\delta_{j}^{*}, \delta_{2}, n-j}(u-v \mid 0) h_{\delta_{1}, \delta_{2}, j}^{(1)}(x, y \mid v)+\int_{0}^{u} h_{\delta_{j}^{*}, \delta_{2}, n-j}(u-s \mid 0) h_{\delta_{1}, \delta_{2}, j}^{(2)}(x, y, v \mid s) d s\right\}, \\
0 \leq v<x ; y>0,
\end{gathered}
$$

depends on lower order discounted densities $h_{\delta_{1}, \delta_{2}, j}^{(2)}(x, y, v \mid s)$ 's for $j=0,1, \ldots, n-1$. (Note that $h_{\delta_{j}^{*}, \delta_{2}, n-j}(u-v \mid 0)$ in the above expression is understood to be 0 when $v \geq u$.) It follows that $(2.10)$ can be reexpressed as

$\nu_{\delta_{12}, n}(u)=\int_{0}^{\infty} \int_{u}^{\infty} w(x, y, u) h_{\delta_{1}, \delta_{2}, n}^{(1)}(x-u, y+u \mid 0) d x d y+\int_{0}^{\infty} \int_{0}^{\infty} \int_{0}^{x} w(x, y, v) \chi_{\delta_{12}, n}^{*}(x, y, v \mid u) d v d x d y$,

where

$$
\chi_{\delta_{12}, n}^{*}(x, y, v \mid u)=\chi_{\delta_{12}, n}(x, y, v \mid u)+h_{\delta_{1}, \delta_{2}, n}^{(2)}(x-u, y+u, v-u \mid 0), \quad 0 \leq v<x ; y>0 .
$$

(The second term above is non-zero only if $v>u$.) Application of (2.15) along with a change of order of integrations leads the integral on the right-hand side of (2.12) to

$$
\begin{aligned}
\int_{0}^{u} a_{\delta_{n}^{*}}(u-t) \nu_{\delta_{12}, n}(t) d t= & \int_{0}^{\infty} \int_{0}^{\infty} \int_{0}^{\min (x, u)} w(x, y, v)\left\{a_{\delta_{n}^{*}}(u-v) h_{\delta_{1}, \delta_{2}, n}^{(1)}(x-v, y+v \mid 0)\right\} d v d x d y \\
& +\int_{0}^{\infty} \int_{0}^{\infty} \int_{0}^{x} w(x, y, v)\left\{\int_{0}^{u} a_{\delta_{n}^{*}}(u-t) \chi_{\delta_{12}, n}^{*}(x, y, v \mid t) d t\right\} d v d x d y .
\end{aligned}
$$

Therefore, putting (2.15) and (2.17) into (2.12) and comparing with (2.4) results in $h_{\delta_{1}, \delta_{2}, n}^{(1)}(x, y \mid u)=$ $h_{\delta_{1}, \delta_{2}, n}^{(1)}(x-u, y+u \mid 0)$ for $x>u$ and $y>0$ (which must be true due to (2.1) and (2.2)), as well as the following Proposition.

Proposition 2 For $n=0,1, \ldots$, the moment-based discounted density $h_{\delta_{1}, \delta_{2}, n}^{(2)}(x, y, v \mid u)$ defined by $(2.3)$ is given by the recursive formula

$$
\begin{aligned}
& h_{\delta_{1}, \delta_{2}, n}^{(2)}(x, y, v \mid u) \\
= & \chi_{\delta_{12}, n}^{*}(x, y, v \mid u)+\frac{1}{1-\alpha_{\delta_{n}^{*}}}\left\{a_{\delta_{n}^{*}}(u-v) h_{\delta_{1}, \delta_{2}, n}^{(1)}(x-v, y+v \mid 0)+\int_{0}^{u} a_{\delta_{n}^{*}}(u-t) \chi_{\delta_{12}, n}^{*}(x, y, v \mid t) d t\right\}, \\
& 0 \leq v<x ; y>0,
\end{aligned}
$$

where $\chi_{\delta_{12, n}}^{*}(x, y, v \mid u)$ is defined by $(2.16)$ together with $(2.14)$, and $a_{\delta_{n}^{*}}(\cdot)$ is the compound geometric density (2.13) (that is understood to be 0 when the argument is non-positive). 
As in Proposition 1, the above Proposition indicates that $h_{\delta_{1}, \delta_{2}, n}^{(2)}(x, y, v \mid 0)$ suffices to characterize $h_{\delta_{1}, \delta_{2}, n}^{(2)}(x, y, v \mid u)$, assuming all the lower order densities are known. Using the same procedure that leads to Proposition 2, the next Corollary follows in a straightforward manner.

Corollary 1 For $n=0,1, \ldots$, the moment-based discounted density $\bar{h}_{\delta_{1}, \delta_{2}, n}(x, y \mid u)$ in $(2.5)$ is given by the recursive formulas

$$
\bar{h}_{\delta_{1}, \delta_{2}, n}(x, y \mid u)=\chi_{\delta_{12}, n}^{* *}(x, y \mid u)+\frac{1}{1-\alpha_{\delta_{n}^{*}}^{*}} \int_{0}^{u} a_{\delta_{n}^{*}}(u-t) \chi_{\delta_{12}, n}^{* *}(x, y \mid t) d t, \quad x, y>0,
$$

where

$$
\chi_{\delta_{12}, n}^{* *}(x, y \mid u)=\sum_{j=0}^{n-1}\left(\begin{array}{c}
n \\
j
\end{array}\right) \int_{0}^{u} h_{\delta_{j}^{*}, \delta_{2}, n-j}(u-s \mid 0) \bar{h}_{\delta_{1}, \delta_{2}, j}(x, y \mid s) d s+\bar{h}_{\delta_{1}, \delta_{2}, n}(x-u, y+u \mid 0), \quad x, y>0 .
$$

The term $\bar{h}_{\delta_{1}, \delta_{2}, n}(x-u, y+u \mid 0)$ appearing in (2.19) is understood to be 0 when $x \leq u$. In addition, $h_{\delta_{1}, \delta_{2}, n}(y \mid u)$ in (2.6) can be obtained from (2.18) in a straightforward manner by integrating over $x$.

Because of the relationship (2.4) at $u=0$, information about $h_{\delta_{1}, \delta_{2}, n}^{(2)}(x, y, v \mid 0)$ can be obtained from $\phi_{\delta_{12}, n}(0)$, which in turn is typically derived starting from another integral equation for $\phi_{\delta_{12}, n}(\cdot)$. Under $U(0)=u$, considering whether ruin occurs upon the first claim event based on the occurrence time $V_{1}$ and the severity $Y_{1}$ results in an integral expression for $\phi_{\delta_{12}, n}(\cdot)$, namely

$\phi_{\delta_{12}, n}(u)=\beta_{\delta_{12}, n}(u)+\int_{0}^{\infty} e^{-\delta_{n}^{*} t}\left\{\int_{0}^{u+c t}\left[\phi_{\delta_{12}, n}(u+c t-y)+\phi_{\delta_{12}, n}^{*}(u+c t, y)\right] p_{t}(y) d y\right\} k(t) d t, \quad u \geq 0$,

where

$$
\begin{aligned}
\beta_{\delta_{12}, n}(u) & =\int_{0}^{\infty} e^{-\delta_{n}^{*} t}\left\{\int_{u+c t}^{\infty} f^{n}(y) w(u+c t, y-u-c t, u) p_{t}(y) d y\right\} k(t) d t \\
& =\int_{u}^{\infty} \int_{0}^{\infty} w(x, y, u) h_{\delta_{1}, \delta_{2}, n}^{(1)}(x, y \mid u) d y d x, \quad u \geq 0,
\end{aligned}
$$

is the contribution by ruin upon the first claim, and

$$
\phi_{\delta_{12}, n}^{*}(x, y)=\sum_{j=0}^{n-1}\left(\begin{array}{l}
n \\
j
\end{array}\right) f^{n-j}(y) \phi_{\delta_{12}, j}(x-y), \quad 0<y \leq x .
$$

We shall proceed by taking Laplace transforms on both sides of (2.20). Define $\widetilde{\phi}_{\delta_{12}, n}(s)=\int_{0}^{\infty} e^{-s u} \phi_{\delta_{12}, n}(u) d u$ and $\widetilde{\beta}_{\delta_{12}, n}(s)=\int_{0}^{\infty} e^{-s u} \beta_{\delta_{12}, n}(u) d u$. Then

$$
\begin{aligned}
\widetilde{\phi}_{\delta_{12}, n}(s)= & \widetilde{\beta}_{\delta_{12}, n}(s)+\int_{0}^{\infty} e^{-s u} \int_{0}^{\infty} e^{-\delta_{n}^{*} t}\left\{\int_{0}^{u+c t} \phi_{\delta_{12}, n}(u+c t-y) p_{t}(y) d y\right\} k(t) d t d u \\
& +\int_{0}^{\infty} e^{-s u} \varphi_{\delta_{12}, n}^{*}\left(u, \delta_{n}^{*}\right) d u,
\end{aligned}
$$

where

$$
\varphi_{\delta_{12}, n}^{*}(u, v)=\int_{0}^{\infty} e^{-v t} \int_{0}^{u+c t} \phi_{\delta_{12}, n}^{*}(u+c t, y) p(t, y) d y d t, \quad u \geq 0 .
$$

Since the triple integral on the right-hand side of (2.24) represents the Laplace transform of a function in the form of Equation (41) in Cheung et al. (2010b), Equation (42) therein is applicable. Hence, it is given by 


$$
\begin{aligned}
& \int_{0}^{\infty} e^{-s u} \int_{0}^{\infty} e^{-\delta_{n}^{*} t}\left\{\int_{0}^{u+c t} \phi_{\delta_{12}, n}(u+c t-y) p_{t}(y) d y\right\} k(t) d t d u \\
= & \widetilde{\phi}_{\delta_{12}, n}(s) \int_{0}^{\infty} e^{-\left(\delta_{n}^{*}-c s\right) t} \widetilde{p}_{t}(s) k(t) d t-\int_{0}^{\infty}\left\{\int_{0}^{c t} e^{-\left(\delta_{n}^{*}-c s\right) t-s x} \int_{0}^{x} \phi_{\delta_{12}, n}(x-y) p_{t}(y) d y d x\right\} k(t) d t \\
= & \widetilde{\phi}_{\delta_{12}, n}(s) \int_{0}^{\infty} e^{-\left(\delta_{n}^{*}-c s\right) t} \widetilde{p}_{t}(s) k(t) d t-\int_{0}^{\infty} e^{-s x} \varphi_{\delta_{12}, n}\left(x, \delta_{n}^{*}-c s\right) d x
\end{aligned}
$$

where

$$
\varphi_{\delta_{12}, n}(x, v)=\int_{x / c}^{\infty} e^{-v t} \int_{0}^{x} \phi_{\delta_{12}, n}(x-y) p(t, y) d y d t, \quad x \geq 0 .
$$

Using (2.26) and defining

$$
\widetilde{\sigma}_{\delta_{12}, n}(s)=\int_{0}^{\infty} e^{-s x}\left[\varphi_{\delta_{12}, n}\left(x, \delta_{n}^{*}-c s\right)-\varphi_{\delta_{12}, n}^{*}\left(x, \delta_{n}^{*}\right)\right] d x
$$

(2.24) can be rewritten as

$$
\widetilde{\phi}_{\delta_{12}, n}(s)=\widetilde{\beta}_{\delta_{12}, n}(s)+\widetilde{\phi}_{\delta_{12}, n}(s) \int_{0}^{\infty} e^{-\left(\delta_{n}^{*}-c s\right) t} \widetilde{p}_{t}(s) k(t) d t-\widetilde{\sigma}_{\delta_{12}, n}(s) .
$$

Rearrangements lead to the following Lemma.

Lemma 1 For $n=0,1, \ldots$, the Laplace transform of $\phi_{\delta_{12}, n}(\cdot)$ admits the representation

$$
\widetilde{\phi}_{\delta_{12}, n}(s)=\frac{\widetilde{\beta}_{\delta_{12, n}}(s)-\widetilde{\sigma}_{\delta_{12}, n}(s)}{1-E\left[e^{-s Y-\left(\delta_{n}^{*}-c s\right) V}\right]},
$$

where

$$
E\left[e^{-s Y-\left(\delta_{n}^{*}-c s\right) V}\right]=\int_{0}^{\infty} e^{-\left(\delta_{n}^{*}-c s\right) t} \widetilde{p}_{t}(s) k(t) d t=\widetilde{p}\left(\delta_{n}^{*}-c s, s\right),
$$

$\widetilde{\beta}_{\delta_{12}, n}(s)$ is the Laplace transform of $(2.21)$, and $\widetilde{\sigma}_{\delta_{12}, n}(s)$ is given by $(2.28)$.

In the above Lemma, while $\widetilde{\beta}_{\delta_{12}, n}(s)$ is completely known, $\widetilde{\sigma}_{\delta_{12}, n}(s)$ in $(2.28)$ depends on $\varphi_{\delta_{12}, n}$ and $\varphi_{\delta_{12, n}}^{*}$. According to (2.27) and (2.25), the quantity $\varphi_{\delta_{12}, n}$ further depends on the Gerber-Shiu function $\phi_{\delta_{12}, n}(\cdot)$ itself; whereas $\varphi_{\delta_{12}, n}^{*}$ depends on the lower order Gerber-Shiu functions $\phi_{\delta_{12}, j}(\cdot)$ 's for $j=0,1, \ldots, n-1$ due to (2.23). In particular, when $n=0$ one has $\varphi_{\delta_{12}, 0}^{*} \equiv \phi_{\delta_{12}, 0}^{*} \equiv 0$, and thus Equation (47) of Cheung et al. (2010b) is retrieved from Lemma 1. Application of Lemma 1 will be given in Section 3.

So far, the analyses in this section hold true for general joint density $p(t, y)$ of the pair $(V, Y)$. Now, if the factorization (1.1) holds, then (2.21) becomes

$$
\beta_{\delta_{12}, n}(u)=\sum_{i=1}^{r} \sum_{j=1}^{m_{i}} \int_{0}^{\infty} e^{-\delta_{n}^{*} t} \gamma_{n, i j}(u+c t, u) k_{i j}(t) d t
$$

where

$$
\gamma_{n, i j}(x, u)=\int_{x}^{\infty} f^{n}(y) w(x, y-x, u) b_{i j}(y) d y=\int_{0}^{\infty} f^{n}(x+y) w(x, y, u) b_{i j}(x+y) d y, \quad x>u .
$$

In addition, in this case $(2.25)$ and $(2.27)$ respectively reduce to

$$
\varphi_{\delta_{12}, n}^{*}(u, v)=\sum_{i=1}^{r} \sum_{j=1}^{m_{i}} \int_{u}^{\infty} \frac{1}{c} e^{-v\left(\frac{x-u}{c}\right)} r_{\delta_{12}, n, i j}^{*}(x) k_{i j}\left(\frac{x-u}{c}\right) d x
$$

and 


$$
\varphi_{\delta_{12}, n}(x, v)=\sum_{i=1}^{r} \sum_{j=1}^{m_{i}} r_{\delta_{12}, n, i j}(x) \int_{x / c}^{\infty} e^{-v t} k_{i j}(t) d t
$$

where

$$
r_{\delta_{12}, n, i j}^{*}(x)=\int_{0}^{x} \phi_{\delta_{12}, n}^{*}(x, y) b_{i j}(y) d y, \quad x \geq 0,
$$

and $r_{\delta_{12}, n, i j}(x)=\int_{0}^{x} \phi_{\delta_{12}, n}(x-y) b_{i j}(y) d y$ for $x \geq 0$.

\section{Coxian interclaim times}

In this section, to identify $\phi_{\delta_{12}, n}(0)$, we consider Coxian distribution for the interclaim times under the dependency structure (1.1) for $(V, Y)$ (see e.g. Willmot and Woo (2012)). Specifically, it is assumed that $k_{i j}(\cdot)$ is an Erlang- $j$ density with scale parameter $\lambda_{i}$, i.e.

$$
k_{i j}(t)=\tau_{i j}(t)=\frac{\lambda_{i}\left(\lambda_{i} t\right)^{j-1} e^{-\lambda_{i} t}}{(j-1) !}, \quad t>0,
$$

and the corresponding Laplace transform is $\widetilde{k}_{i j}(s)=\widetilde{\tau}_{i j}(s)=\left[\lambda_{i} /\left(\lambda_{i}+s\right)\right]^{j}$. In this case, the marginal density of the interclaim time is

$$
k(t)=\sum_{i=1}^{r} \sum_{j=1}^{m_{i}} \widetilde{b}_{i j}(0) \tau_{i j}(t), \quad t>0,
$$

and its Laplace transform can be expressed as $\widetilde{k}(s)=\eta(s) / \prod_{i=1}^{r}\left(\lambda_{i}+s\right)^{m_{i}}$, where $\prod_{i=1}^{r}\left(\lambda_{i}+s\right)^{m_{i}}$ is a polynomial in $s$ of degree $m=\sum_{i=1}^{r} m_{i}$ and $\eta(s)$ is also a polynomial in $s$ but of degree $n-1$ or less. Clearly, $k(\cdot)$ in $(3.2)$ corresponds to the density of a Coxian distribution (and $\widetilde{b}_{i j}(0)$ 's are not necessarily positive). Without loss of generality it is assumed that $\lambda_{i}$ 's are all distinct. Some examples of dependency structures that are special cases of the above assumptions are given in Willmot and Woo (2012, Section $5)$.

It is noted that $\varphi_{\delta_{12}, n}(x, v)$ defined in (2.32) is structurally identical to Equation (13) in Willmot and Woo (2012), who studied the Gerber-Shiu function $\phi_{\delta_{12}, 0}(u)$ (i.e. the generalized Gerber-Shiu function including $R$ but without any moment-based component). Therefore, details of the derivations arising from this function will be mostly omitted in the upcoming analysis. Instead, we focus more on functions that are related to the term $\varphi_{\delta_{12}, n}^{*}(u, v)$ in (2.25) (or (2.31)), which is new due to the introduction of the moment-based component $Z_{\delta_{2}}^{n}(\tau)$ in (1.3). Under (1.2) and (3.1), the Laplace transform of $\phi_{\delta_{12}, n}(\cdot)$ in Lemma 1 becomes

$$
\widetilde{\phi}_{\delta_{12}, n}(s)=\frac{\widetilde{\beta}_{\delta_{12}, n}(s)-\widetilde{\sigma}_{\delta_{12}, n}(s)}{1-\sum_{i=1}^{r} \sum_{j=1}^{m_{i}}\left(\frac{\lambda_{i}}{\lambda_{i}+\delta_{n}^{*}-c s}\right)^{j} \widetilde{b}_{i j}(s)} .
$$

We proceed by studying the term $\widetilde{\sigma}_{\delta_{12}, n}(s)$ in (2.28). From Willmot and Woo (2012, Equation (27)), the first term on the right-hand side of $(2.28)$ is given by

$$
\int_{0}^{\infty} e^{-s x} \varphi_{\delta_{12}, n}\left(x, \delta_{n}^{*}-c s\right) d x=\sum_{i=1}^{r} \sum_{j=1}^{m_{i}} \frac{\theta_{\delta_{12, n, i j}}}{\left(\lambda_{i}+\delta_{n}^{*}-c s\right)^{j}}
$$

where $\theta_{\delta_{12}, n, i j}=\sum_{k=j}^{m_{i}} \frac{\lambda_{i}^{k} \widetilde{r}_{\delta_{12}, n, i k}^{(k-j)}\left(\frac{\lambda_{i}+\delta_{n}^{*}}{c}\right)}{(-c)^{k-j}(k-j) !}$, with $\widetilde{r}_{\delta_{12}, n, i k}^{(j)}(s)=\int_{0}^{\infty}(-x)^{j} e^{-s x} r_{\delta_{12}, n, i k}(x) d x$. For the second term in (2.28), with (3.1) we first evaluate (2.31) as 


$$
\varphi_{\delta_{12}, n}^{*}(u, v)=\sum_{i=1}^{r} \sum_{j=1}^{m_{i}}\left(\frac{\lambda_{i}}{\lambda_{i}+v}\right)^{j} \int_{u}^{\infty} \frac{1}{c} r_{\delta_{12}, n, i j}^{*}(x) \tau_{i_{v} j}\left(\frac{x-u}{c}\right) d x,
$$

where (with a slight abuse of notation) $\tau_{i_{v} j}(t)$ is an Erlang- $j$ density with scale parameter $\lambda_{i}+v$. Define $\widetilde{r}_{\delta_{12}, n, i j}^{*}(s)=\int_{0}^{\infty} e^{-s x} r_{\delta_{12}, n, i j}^{*}(x) d x$. Then, taking Laplace transform followed by a change of order of integrations and a change of variable $t=(x-u) / c$ results in

$$
\begin{aligned}
& \int_{0}^{\infty} e^{-s u} \varphi_{\delta_{12}, n}^{*}(u, v) d u=\sum_{i=1}^{r} \sum_{j=1}^{m_{i}}\left(\frac{\lambda_{i}}{\lambda_{i}+v}\right)^{j} \int_{0}^{\infty} e^{-s x} r_{\delta_{12}, n, i j}^{*}(x) \int_{0}^{x / c} e^{c s t} \tau_{i_{v} j}(t) d t d x \\
= & \sum_{i=1}^{r} \sum_{j=1}^{m_{i}}\left\{\widetilde{\tau}_{i j}(v-c s) \widetilde{r}_{\delta_{12}, n, i j}^{*}(s)-\left(\frac{\lambda_{i}}{\lambda_{i}+v}\right)^{j} \int_{0}^{\infty} e^{-s x} r_{\delta_{12}, n, i j}^{*}(x) \int_{x / c}^{\infty} e^{c s t} \tau_{i_{v} j}(t) d t d x\right\} \\
= & \sum_{i=1}^{r} \sum_{j=1}^{m_{i}} \widetilde{\tau}_{i j}(v-c s) \widetilde{r}_{\delta_{12}, n, i j}^{*}(s)-\sum_{i=1}^{r} \sum_{j=1}^{m_{i}} \frac{\theta_{\delta_{12}, n, i j}^{*}(v)}{\left(\lambda_{i}+v-c s\right)^{j}},
\end{aligned}
$$

where the last line follows from Willmot and Woo (2012, Equation (27)), with $\theta_{\delta_{12}, n, i j}^{*}(v)=$ $\sum_{k=j}^{m_{i}} \frac{\lambda_{i}^{k} \widetilde{r}_{\delta_{12}, n, i k}^{*(k-j)}\left(\frac{\lambda_{i}+v}{c}\right)}{(-c)^{k-j}(k-j) !}$ and $\widetilde{r}_{\delta_{12}, n, i k}^{*(j)}(s)=\int_{0}^{\infty}(-x)^{j} e^{-s x} r_{\delta_{12}, n, i k}^{*}(x) d x$. Application of (3.4) and (3.5) leads $(2.28)$ to

$$
\widetilde{\sigma}_{\delta_{12}, n}(s)=\sum_{i=1}^{r} \sum_{j=1}^{m_{i}} \frac{\theta_{\delta_{12}, n, i j}^{* *}}{\left(\lambda_{i}+\delta_{n}^{*}-c s\right)^{j}}-\widetilde{\beta}_{\delta_{12}, n}^{*}(s),
$$

where $\theta_{\delta_{12}, n, i j}^{* *}=\theta_{\delta_{12}, n, i j}+\theta_{\delta_{12}, n, i j}^{*}\left(\delta_{n}^{*}\right)$, and

$$
\widetilde{\beta}_{\delta_{12}, n}^{*}(s)=\sum_{i=1}^{r} \sum_{j=1}^{m_{i}} \widetilde{\tau}_{i j}\left(\delta_{n}^{*}-c s\right) \widetilde{r}_{\delta_{12}, n, i j}^{*}(s) .
$$

Consequently, (3.3) can be rewritten as

$$
\widetilde{\phi}_{\delta_{12}, n}(s)=\frac{\widetilde{\beta}_{\delta_{12}, n}^{* *}(s)-\widetilde{\sigma}_{\delta_{12}, n}^{*}(s)}{1-\sum_{i=1}^{r} \sum_{j=1}^{m_{i}}\left(\frac{\lambda_{i}}{\lambda_{i}+\delta_{n}^{*}-c s}\right)^{j} \widetilde{b}_{i j}(s)},
$$

where

$$
\widetilde{\beta}_{\delta_{12}, n}^{* *}(s)=\widetilde{\beta}_{\delta_{12}, n}(s)+\widetilde{\beta}_{\delta_{12}, n}^{*}(s)
$$

and

$$
\tilde{\sigma}_{\delta_{12}, n}^{*}(s)=\widetilde{\sigma}_{\delta_{12}, n}(s)+\widetilde{\beta}_{\delta_{12}, n}^{*}(s)=\sum_{i=1}^{r} \sum_{j=1}^{m_{i}} \frac{\theta_{\delta_{12}, n, i j}^{* *}}{\left(\lambda_{i}+\delta_{n}^{*}-c s\right)^{j}}=\frac{q_{\delta_{12}, n}(s)}{\prod_{i=1}^{r}\left(\lambda_{i}+\delta_{n}^{*}-c s\right)^{m_{i}}},
$$

with $q_{\delta_{12}, n}(s)=\left\{\prod_{i=1}^{r}\left(\lambda_{i}+\delta_{n}^{*}-c s\right)^{m_{i}}\right\} \sum_{i=1}^{r} \sum_{j=1}^{m_{i}} \frac{\theta_{\delta_{12}, n, i j}^{* *}}{\left(\lambda_{i}+\delta_{n}^{*}-c s\right)^{j}}$ being a polynomial in $s$ of degree at most $m-1$.

As $\tilde{\sigma}_{\delta_{12}, n}^{*}(s)$ is expressed as a ratio of two polynomials in $(3.10)$, the procedure to obtain $\phi_{\delta_{12}, n}(0)$ from $\widetilde{\phi}_{\delta_{12}, n}(s)$ using (3.8) is similar to the arguments that lead to $\widetilde{\phi}_{\delta_{12}, 0}(s)$ in Willmot and Woo $(2012$, Section 3). Therefore, applying Equations (32) and (33) therein (obtained from the theory of Lagrange polynomials and initial value theorem for Laplace transforms) yields

$$
\phi_{\delta_{12}, n}(0)=\beta_{\delta_{12}, n}^{* *}(0)+\sum_{k=1}^{m} A_{k, n} \widetilde{\beta}_{\delta_{12}, n}^{* *}\left(\rho_{k, n}\right),
$$


where $\beta_{\delta_{12}, n}^{* *}(0)=\lim _{s \rightarrow \infty} s \widetilde{\beta}_{\delta_{12}, n}^{* *}(s)$ and

$$
A_{k, n}=\frac{\prod_{i=1}^{r}\left(\frac{\lambda_{i}+\delta_{n}^{*}}{c}-\rho_{k, n}\right)^{m_{i}}}{\prod_{j=1, j \neq k}^{m}\left(\rho_{j, n}-\rho_{k, n}\right)} .
$$

In addition, for each fixed $n=0,1, \ldots$, the quantities $\left\{\rho_{k, n}\right\}_{k=1}^{m}$ are the $m$ roots of the Lundberg's equation (in $\xi$ )

$$
\widetilde{p}\left(\delta_{n}^{*}-c \xi, \xi\right)=\sum_{i=1}^{r} \sum_{j=1}^{m_{i}}\left(\frac{\lambda_{i}}{\lambda_{i}+\delta_{n}^{*}-c \xi}\right)^{j} \widetilde{b}_{i j}(\xi)=1
$$

with non-negative real parts. These roots are typically assumed to be distinct (see Remark 1). For notational convenience, the dependence of $\left\{\rho_{k, n}\right\}_{k=1}^{m}$ and $\left\{A_{k, n}\right\}_{k=1}^{m}$ on $\delta_{1}$ and $\delta_{2}$ has been suppressed. Note that $\widetilde{\beta}_{\delta_{12}, n}^{* *}(\cdot)$ defined by $(3.9)$ comprises two components. While $\widetilde{\beta}_{\delta_{12}, n}(\cdot)$ is simply the Laplace transform of the known quantity $(2.22)$, the component $\widetilde{\beta}_{\delta_{12}, n}^{*}(\cdot)$ in $(3.7)$ depends on the lower order Gerber-Shiu functions $\phi_{\delta_{12}, j}(\cdot)$ 's for $j=0,1, \ldots, n-1$ because of (2.23) and (2.33). From (3.7), one has $\lim _{s \rightarrow \infty} s \widetilde{\beta}_{\delta_{12}, n}^{*}(s)=0$ since $\lim _{s \rightarrow \infty} \widetilde{\tau}_{i j}\left(\delta_{n}^{*}-c s\right)=0$ by analytic continuation of Laplace transform and $\lim _{s \rightarrow \infty} s \widetilde{r}_{\delta_{12}, n, i j}^{*}(s)=r_{\delta_{12}, n, i j}^{*}(0)=0$ according to (2.33). Thus, multiplying both sides of (3.9) by $s$ followed by letting $s \rightarrow \infty$ along with the use of (2.22) yields

$$
\beta_{\delta_{12}, n}^{* *}(0)=\beta_{\delta_{12}, n}(0)=\int_{0}^{\infty} \int_{0}^{\infty} w(x, y, 0) h_{\delta_{1}, \delta_{2}, n}^{(1)}(x, y \mid 0) d y d x .
$$

With (3.13), comparison of (2.4) at $u=0$ with (3.11) leads to

$\int_{0}^{\infty} \int_{0}^{\infty} \int_{0}^{x} w(x, y, v) h_{\delta_{1}, \delta_{2}, n}^{(2)}(x, y, v \mid 0) d v d x d y=\sum_{k=1}^{m} A_{k, n} \widetilde{\beta}_{\delta_{12}, n}^{* *}\left(\rho_{k, n}\right)=\sum_{k=1}^{m} A_{k, n}\left[\widetilde{\beta}_{\delta_{12}, n}\left(\rho_{k, n}\right)+\widetilde{\beta}_{\delta_{12}, n}^{*}\left(\rho_{k, n}\right)\right]$,

where the last equality follows from (3.9). The identification of the moment-based discounted density $h_{\delta_{1}, \delta_{2}, n}^{(2)}(x, y, v \mid 0)$ can be done via the above equation as follows. Utilizing (2.22), we first obtain

$$
\widetilde{\beta}_{\delta_{12}, n}\left(\rho_{k, n}\right)=\int_{0}^{\infty} \int_{0}^{\infty} \int_{0}^{x} w(x, y, v)\left\{h_{\delta_{1}, \delta_{2}, n}^{(1)}(x, y \mid v) e^{-\rho_{k, n} v}\right\} d v d x d y .
$$

Next, putting $s=\rho_{k, n}$ into (3.7) and using (2.23) and (2.33) give rise to

$$
\widetilde{\beta}_{\delta_{12}, n}^{*}\left(\rho_{k, n}\right)=\sum_{i=1}^{r} \sum_{j=1}^{m_{i}} \widetilde{\tau}_{i j}\left(\delta_{n}^{*}-c \rho_{k, n}\right) \sum_{l=0}^{n-1}\left(\begin{array}{l}
n \\
l
\end{array}\right) \int_{0}^{\infty} e^{-\rho_{k, n} v} \int_{0}^{v} f^{n-l}(y) \phi_{\delta_{12}, l}(v-y) b_{i j}(y) d y d v .
$$

Now we pay attention to the double integral, which can regarded as the Laplace transform (with argument $\left.\rho_{k, n}\right)$ of a convolution. In particular, we define, for $n=1,2, \ldots$,

$$
\widetilde{b}_{n, i j}^{f}(s)=\int_{0}^{\infty} e^{-s y} f^{n}(y) b_{i j}(y) d y
$$

Then, by applying (2.4) we arrive at

$$
\begin{aligned}
& \int_{0}^{\infty} e^{-\rho_{k, n} v} \int_{0}^{v} f^{n-l}(y) \phi_{\delta_{12}, l}(v-y) b_{i j}(y) d y d v=\widetilde{b}_{n-l, i j}^{f}\left(\rho_{k, n}\right) \int_{0}^{\infty} e^{-\rho_{k, n} v} \phi_{\delta_{12}, l}(v) d v \\
= & \widetilde{b}_{n-l, i j}^{f}\left(\rho_{k, n}\right)\left(\int_{0}^{\infty} \int_{0}^{\infty} \int_{0}^{x} w(x, y, v)\left\{e^{-\rho_{k, n} v} h_{\delta_{1}, \delta_{2}, l}^{(1)}(x, y \mid v)+\int_{0}^{\infty} e^{-\rho_{k, n} u} h_{\delta_{1}, \delta_{2}, l}^{(2)}(x, y, v \mid u)\right\} d u d v d x d y\right) .
\end{aligned}
$$

Combining (3.14)-(3.16) and (3.18), we can find $h_{\delta_{1}, \delta_{2}, n}^{(2)}(x, y, v \mid 0)$ in the following Proposition. 
Proposition 3 Assume that the joint density of $(V, Y)$ follows the dependency structure (1.1) with $k_{i j}(\cdot)$ given by (3.1). For $n=0,1, \ldots$, the moment-based discounted density $h_{\delta_{1}, \delta_{2}, n}^{(2)}(x, y, v \mid 0)$ defined in $(2.3)$ with zero initial surplus can be obtained recursively via

$$
\begin{aligned}
& h_{\delta_{1}, \delta_{2}, n}^{(2)}(x, y, v \mid 0)=h_{\delta_{1}, \delta_{2}, n}^{(1)}(x, y \mid v) \sum_{k=1}^{m} A_{k, n} e^{-\rho_{k, n} v} \\
& +\sum_{k=1}^{m} A_{k, n} \sum_{i=1}^{r} \sum_{j=1}^{m_{i}} \widetilde{\tau}_{i j}\left(\delta_{n}^{*}-c \rho_{k, n}\right) \sum_{l=0}^{n-1}\left(\begin{array}{c}
n \\
l
\end{array}\right) \widetilde{b}_{n-l, i j}^{f}\left(\rho_{k, n}\right)\left\{e^{-\rho_{k, n} v} h_{\delta_{1}, \delta_{2}, l}^{(1)}(x, y \mid v)+\int_{0}^{\infty} e^{-\rho_{k, n} u} h_{\delta_{1}, \delta_{2}, l}^{(2)}(x, y, v \mid u) d u\right\} \\
& 0 \leq v<x ; y>0
\end{aligned}
$$

where $\widetilde{b}_{n-l, i j}^{f}(\cdot)$ is defined in $(3.17)$.

In Proposition 3, for each fixed $n=0,1, \ldots$, the density $h_{\delta_{1}, \delta_{2}, n}^{(2)}(x, y, v \mid 0)$ is expressed in terms of the lower order densities $h_{\delta_{1}, \delta_{2}, l}^{(2)}\left(x, y, v \mid u\right.$ )'s for $l=0,1, \ldots, n-1$ (with $h^{(1)}$ known explicitly via (2.1) and $(2.2))$. Because $h_{\delta_{1}, \delta_{2}, 0}^{(1)}(x, y \mid u)$ and $h_{\delta_{1}, \delta_{2}, 0}^{(2)}(x, y, v \mid u)$ are traditional discounted densities (i.e. without any moment-based components) that do not depend on $\delta_{2}$, we define $h_{1, \delta_{1}}(x, y \mid u)=h_{\delta_{1}, \delta_{2}, 0}^{(1)}(x, y \mid u)$ and $h_{2, \delta_{1}}(x, y, v \mid u)=h_{\delta_{1}, \delta_{2}, 0}^{(2)}(x, y, v \mid u)$. Then, at the starting point $n=0,(3.19)$ reduces to

$$
h_{2, \delta_{1}}(x, y, v \mid 0)=h_{1, \delta_{1}}(x, y \mid v) \sum_{k=1}^{m} A_{k, 0} e^{-\rho_{k, 0} v}, \quad 0 \leq v<x ; y>0,
$$

which is consistent with Willmot and Woo (2012, Equation (34)). According to Proposition 2, this is sufficient to characterize $h_{2, \delta_{1}}\left(x, y, v \mid u\right.$ ) (for $u \geq 0$ ), which can in turn be used to determine $h_{\delta_{1}, \delta_{2}, 1}^{(2)}(x, y, v \mid 0)$ via application of Proposition 3 when $n=1$. Then we can continue to utilize Propositions 2 and 3 repetitively until $h_{\delta_{1}, \delta_{2}, n}^{(2)}(x, y, v \mid 0)$ is obtained. If our goal is to calculate $h_{\delta_{1}, \delta_{2}, n}^{(2)}(x, y, v \mid u)$, then one can apply Proposition 2 once more. But if we are interested in the Gerber-Shiu function $\phi_{\delta_{12}, n}(u)$ defined by (1.3), then one can either use Proposition 1 or the integral representation (2.4) with the help of $h_{\delta_{1}, \delta_{2}, n}^{(2)}(x, y, v \mid u)$.

Remark 1 Sometimes it is possible that the roots $\left\{\rho_{k, n}\right\}_{k=1}^{m}$ are not distinct. Under such a rare situation, practically one can slightly alter a model parameter (such as $\delta_{1}, \delta_{2}$ or the premium rate $c$ ) so that there will no longer be multiple roots. Then the ruin-related quantities in question could be approximated or bounded by their counterparts in a model with distinct roots. Interested readers are referred to D'Auria et al. (2010), Labbé et al. (2011), and Ji and Zhang (2012) for the detailed treatment of risk processes with multiple Lundberg's roots.

Example 1 Consider the classical compound Poisson risk model with time-independent claims (i.e. $r=1$ and $m_{1}=1$ in (1.1) and (3.1)). For notational convenience, we let $\lambda_{1}=\lambda$ and $\rho_{1,1}=\rho$, and the claim density is $b_{11}(\cdot) \equiv p(\cdot)$. In this case, $(2.2)$ is given by $h_{\delta_{1}, \delta_{2}, n}^{(1)}(x, y \mid u)=\frac{\lambda}{c} e^{-\left(\frac{\lambda+\delta_{1}+n \delta_{2}}{c}\right)(x-u)} f^{n}(x+y) p(x+y)$ for $x>u$ and $y>0$. When $n=1,(3.19)$ reduces to

$$
\begin{aligned}
h_{\delta_{1}, \delta_{2}, 1}^{(2)}(x, y, v \mid 0)= & h_{\delta_{1}, \delta_{2}, 1}^{(1)}(x, y \mid v)\left(\frac{\lambda+\delta_{1}+\delta_{2}}{c}-\rho\right) e^{-\rho v} \\
& +\frac{\lambda}{c} \widetilde{(f \cdot p)}(\rho)\left\{e^{-\rho v} h_{1, \delta_{1}}(x, y \mid v)+\int_{0}^{\infty} e^{-\rho u} h_{2, \delta_{1}}(x, y, v \mid u) d u\right\}, \quad 0 \leq v<x ; y>0,
\end{aligned}
$$


where $\widetilde{(f \cdot p)}(\rho)=\int_{0}^{\infty} e^{-\rho y} f(y) p(y) d y$. The quantity $h_{2, \delta_{1}}(x, y, v \mid u)$ has been derived by Cheung et al. (2010a, Corollary 3.1).

For the rest of this section, we consider the special case of $(1.3)$ when $w(x, y, v)=\bar{w}(x, y)$, i.e. the penalty function does not depend on the variable $R$. Such a Gerber-Shiu function is given by

$$
\bar{\phi}_{\delta_{12}, n}(u)=E\left[e^{-\delta_{1} \tau} Z_{\delta_{2}}^{n}(\tau) \bar{w}\left(U\left(\tau^{-}\right),|U(\tau)|\right) I\{\tau<\infty\} \mid U(0)=u\right], \quad u \geq 0,
$$

which is an extension of the classical Gerber-Shiu function further involving the $n$-th moment of the discounted aggregate claim costs until ruin. Similarly, the special cases of some related functions will be denoted by adding a bar ' - ' on them. As we shall see, some of the previous results can be simplified. Again we shall apply Lemma 1 (or Equation (3.3)) and identify the corresponding $\widetilde{\bar{\beta}}_{\delta_{12}, n}(s)=\int_{0}^{\infty} e^{-s u} \bar{\beta}_{\delta_{12}, n}(u) d u$ and $\widetilde{\bar{\sigma}}_{\delta_{12}, n}(s)$ under the penalty $w(x, y, v)=\bar{w}(x, y)$. Then, with the distributional assumption $k_{i j}(t)=\tau_{i j}(t)$ in (3.1), (2.29) reduces to

$$
\bar{\beta}_{\delta_{12}, n}(u)=\sum_{i=1}^{r} \sum_{j=1}^{m_{i}} \int_{0}^{\infty} e^{-\delta_{n}^{*} t} \bar{\gamma}_{n, i j}(u+c t) \tau_{i j}(t) d t, \quad u \geq 0,
$$

where

$$
\bar{\gamma}_{n, i j}(x)=\int_{x}^{\infty} f^{n}(y) \bar{w}(x, y-x) b_{i j}(y) d y=\int_{0}^{\infty} f^{n}(x+y) \bar{w}(x, y) b_{i j}(x+y) d y, \quad x>0
$$

from (2.30) no longer depends on $u$. For later use, we also define the corresponding Laplace transform

$$
\widetilde{\bar{\gamma}}_{n, i j}(s)=\int_{0}^{\infty} e^{-s x} \bar{\gamma}_{n, i j}(x) d x=\int_{0}^{\infty} \int_{0}^{\infty} \bar{w}(x, y) e^{-s x} f^{n}(x+y) b_{i j}(x+y) d x d y .
$$

Along the same lines as in Willmot and Woo (2012, p.138), it follows that

$$
\widetilde{\bar{\beta}}_{\delta_{12}, n}(s)=\sum_{i=1}^{r} \sum_{j=1}^{m_{i}} \widetilde{\tau}_{i j}\left(\delta_{n}^{*}-c s\right) \widetilde{\bar{\gamma}}_{n, i j}(s)-\sum_{i=1}^{r} \sum_{j=1}^{m_{i}} \frac{\vartheta_{\delta_{12, n, i j}}}{\left(\lambda_{i}+\delta_{n}^{*}-c s\right)^{j}},
$$

with $\vartheta_{\delta_{12}, n, i j}$ 's being constants. As for $\widetilde{\bar{\sigma}}_{\delta_{12}, n}(s),(3.6)$ in the present context becomes

$$
\widetilde{\bar{\sigma}}_{\delta_{12}, n}(s)=\sum_{i=1}^{r} \sum_{j=1}^{m_{i}} \frac{\bar{\theta}_{\delta_{12}, n, i j}^{* *}}{\left(\lambda_{i}+\delta_{n}^{*}-c s\right)^{j}}-\widetilde{\bar{\beta}}_{\delta_{12}, n}^{*}(s),
$$

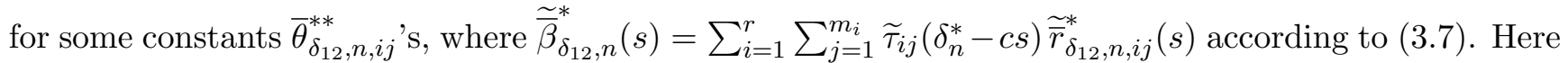
$\widetilde{\bar{r}}_{\delta_{12}, n, i j}^{*}(s)$ follows the trivial definition $\widetilde{\bar{r}}_{\delta_{12}, n, i j}^{*}(s)=\int_{0}^{\infty} e^{-s x} \bar{r}_{\delta_{12}, n, i j}^{*}(x) d x$ with $\bar{r}_{\delta_{12}, n, i j}^{*}(x)=$ $\int_{0}^{x} \bar{\phi}_{\delta_{12}, n}^{*}(x, y) b_{i j}(y) d y$ and $\bar{\phi}_{\delta_{12}, n}^{*}(x, y)=\sum_{j=0}^{n-1}\left(\begin{array}{c}n \\ j\end{array}\right) f^{n-j}(y) \bar{\phi}_{\delta_{12}, j}(x-y)$ because of $(2.33)$ and $(2.23)$ respectively. Define $\widetilde{\bar{\phi}}_{\delta_{12}, n}(s)=\int_{0}^{\infty} e^{-s u} \bar{\phi}_{\delta_{12}, n}(u) d u$. When $w(x, y, v)=\bar{w}(x, y)$, substitution of $(3.22)$ and (3.23) into (3.3) yields

$$
\widetilde{\bar{\phi}}_{\delta_{12}, n}(s)=\frac{\widetilde{\zeta}_{\delta_{12}, n}(s)-\widetilde{\varpi}_{\delta_{12}, n}(s)}{1-\sum_{i=1}^{r} \sum_{j=1}^{m_{i}}\left(\frac{\lambda_{i}}{\lambda_{i}+\delta_{n}^{*}-c s}\right)^{j} \widetilde{b}_{i j}(s)} .
$$


where

$$
\widetilde{\zeta}_{\delta_{12}, n}(s)=\sum_{i=1}^{r} \sum_{j=1}^{m_{i}} \widetilde{\tau}_{i j}\left(\delta_{n}^{*}-c s\right) \widetilde{\bar{\gamma}}_{n, i j}(s)+\widetilde{\widetilde{\beta}}_{\delta_{12}, n}^{*}(s)
$$

and

$$
\widetilde{\varpi}_{\delta_{12}, n}(s)=\sum_{i=1}^{r} \sum_{j=1}^{m_{i}} \frac{\vartheta_{\delta_{12}, n, i j}^{*}}{\left(\lambda_{i}+\delta_{n}^{*}-c s\right)^{j}}=\frac{q_{\delta_{12}, n}^{*}(s)}{\prod_{i=1}^{r}\left(\lambda_{i}+\delta_{n}^{*}-c s\right)^{m_{i}}},
$$

with $\vartheta_{\delta_{12}, n, i j}^{*}=\vartheta_{\delta_{12}, n, i j}+\bar{\theta}_{\delta_{12}, n, i j}^{* *}$ and $q_{\delta_{12}, n}^{*}(s)$ being a polynomial in $s$ of degree at most $m-1$. By applying the same arguments resulting in (3.11) and noting that $\lim _{s \rightarrow \infty} s \widetilde{\zeta}_{\delta_{12}, n}(s)=0$ as $\widetilde{\tau}_{i j}(s)=\left[\lambda_{i} /\left(\lambda_{i}+s\right)\right]^{j}$, one finds

$$
\bar{\phi}_{\delta_{12}, n}(0)=\sum_{k=1}^{m} A_{k, n} \widetilde{\zeta}_{\delta_{12}, n}\left(\rho_{k, n}\right)
$$

where $A_{k, n}$ 's are given by (3.12). Concerning $\widetilde{\zeta}_{\delta_{12}, n}(\cdot)$ in $(3.24)$, we note that $\widetilde{\bar{\gamma}}_{n, i j}(\cdot)$ is explicitly known via (3.20) whereas $\widetilde{\bar{\beta}}_{\delta_{12, n}}^{*}(\cdot)$ depends on the lower order Gerber-Shiu functions $\bar{\phi}_{\delta_{12}, j}(\cdot)$ 's for $j=0,1, \ldots, n-1$.

From (2.4) along with $w(x, y, v)=\bar{w}(x, y)$, one can write

$$
\bar{\phi}_{\delta_{12}, n}(u)=\int_{0}^{\infty} \int_{0}^{\infty} \bar{w}(x, y) \bar{h}_{\delta_{1}, \delta_{2}, n}(x, y \mid u) d x d y, \quad u \geq 0
$$

where $\bar{h}_{\delta_{1}, \delta_{2}, n}(x, y \mid u)$ is given by (2.5). We shall identify $\bar{h}_{\delta_{1}, \delta_{2}, n}(x, y \mid 0)$. Resembling (3.16) and (3.18), it is found that

$\widetilde{\widetilde{\beta}}_{\delta_{12}, n}^{*}\left(\rho_{k, n}\right)=\sum_{i=1}^{r} \sum_{j=1}^{m_{i}} \widetilde{\tau}_{i j}\left(\delta_{n}^{*}-c \rho_{k, n}\right) \sum_{l=0}^{n-1}\left(\begin{array}{l}n \\ l\end{array}\right) \widetilde{b}_{n-l, i j}^{f}\left(\rho_{k, n}\right) \int_{0}^{\infty} \int_{0}^{\infty} \bar{w}(x, y) \int_{0}^{\infty} e^{-\rho_{k, n} u} \bar{h}_{\delta_{1}, \delta_{2}, l}(x, y \mid u) d u d x d y$.

Application of the above equation and (3.21) with $s=\rho_{k, n}$ to (3.24) followed by comparing (3.25) with (3.26) at $u=0$ leads to following Proposition.

Proposition 4 Assume that the joint density of $(V, Y)$ follows the dependency structure (1.1) with $k_{i j}(\cdot)$ given by (3.1). For $n=0,1, \ldots$, the moment-based discounted density $\bar{h}_{\delta_{1}, \delta_{2}, n}(x, y \mid 0)$ in (2.5) with zero initial surplus can be obtained recursively via

$$
\begin{aligned}
\bar{h}_{\delta_{1}, \delta_{2}, n}(x, y \mid 0)=\sum_{k=1}^{m} A_{k, n} \sum_{i=1}^{r} & \sum_{j=1}^{m_{i}} \widetilde{\tau}_{i j}\left(\delta_{n}^{*}-c \rho_{k, n}\right)\left\{e^{-\rho_{k, n} x} f^{n}(x+y) b_{i j}(x+y)\right. \\
& \left.+\sum_{l=0}^{n-1}\left(\begin{array}{l}
n \\
l
\end{array}\right) \widetilde{b}_{n-l, i j}^{f}\left(\rho_{k, n}\right) \int_{0}^{\infty} e^{-\rho_{k, n} u} \bar{h}_{\delta_{1}, \delta_{2}, l}(x, y \mid u) d u\right\}, \quad x, y>0 .
\end{aligned}
$$

The density $h_{\delta_{1}, \delta_{2}, n}(y \mid 0)$ follows immediately by integrating over $x$ thanks to $(2.6)$.

The starting point $n=0$ of (3.27) represents the traditional discounted joint density of $\left(U^{-},|U|\right)$ with zero initial surplus, and it agrees with Willmot and Woo (2012, Equation (41)). Following the comments made after Proposition 3, (2.18) and (2.19) in Corollary 1 along with (3.27) in Proposition 4 lead to a full characterization of the recursive evaluation of $\bar{h}_{\delta_{1}, \delta_{2}, n}(x, y \mid u)$ for $u \geq 0$. 


\section{Combination of exponentials claims: Discounted claims until ruin}

In this section, the joint density (1.1) for $(V, Y)$ is still assumed. However, in contrast to Section 3 in which distributional assumption on $k_{i j}(\cdot)$ is made, we shall assume specific form of $b_{i j}(\cdot)$ pertaining to the claim severity (see (4.3)). As suggested by Willmot (2007), the study of Gerber-Shiu function in risk processes with general interclaim times typically starts with the identification of the form of the 'first drop' density $h_{\delta_{1}, \delta_{2}, n}(y \mid 0)$ as a function of $y$ via some probabilistic arguments. For ruin of the surplus process by claims subsequent to the first, we shall look at the joint density of $\left(\tau, Z_{\delta_{2}}, U^{-},|U|, R\right)$ with zero initial surplus, namely $g_{2, \delta_{2}}(t, z, x, y, v \mid 0)$, defined at the beginning of Section 2 . Let $g_{\delta_{2}}^{*}(t, z, x, v \mid 0)$ be the joint density of the quadruple $\left(\tau, \sum_{i=1}^{N(\tau)-1} e^{-\delta_{2} T_{i}} f\left(Y_{i}\right), U^{-}, R\right)$ at $(t, z, x, v)$ for ruin occurring upon subsequent claims, given $U(0)=0$. Combining the arguments leading to Cheung (2013, Equation (2.4)) and Landriault et al. (2013, Equation (7)), we arrive at, for $0 \leq v<x, y>0, t>x / c$, and $z>e^{-\delta_{2} t} f(x+y)$,

$$
g_{2, \delta_{2}}(t, z, x, y, v \mid 0)=g_{\delta_{2}}^{*}\left(t, z-e^{-\delta_{2} t} f(x+y), x, v \mid 0\right) \frac{g_{1}(x, y \mid v)}{\int_{0}^{\infty} g_{1}(x, s \mid v) d s},
$$

where $g_{1}$ is defined in (2.1). Under (1.1), application of (2.1)-(2.3) and (4.1) to (2.6) yields

$$
\begin{aligned}
& h_{\delta_{1}, \delta_{2}, n}(y \mid 0) \\
= & \int_{0}^{\infty} e^{-\delta_{n}^{*}\left(\frac{x}{c}\right)} f^{n}(x+y) g_{1}(x, y \mid 0) d x \\
& +\int_{0}^{\infty} \int_{0}^{x} \int_{x / c}^{\infty} \int_{e^{-\delta_{2} t} f(x+y)}^{\infty} e^{-\delta_{1} t} z^{n} g_{\delta_{2}}^{*}\left(t, z-e^{-\delta_{2} t} f(x+y), x, v \mid 0\right) \frac{g_{1}(x, y \mid v)}{\int_{0}^{\infty} g_{1}(x, s \mid v) d s} d z d t d v d x \\
= & \sum_{i=1}^{r} \sum_{j=1}^{m_{i}} \int_{0}^{\infty} e^{-\frac{\delta_{n}^{*}}{c} x} f^{n}(x+y)\left\{\frac{1}{c} k_{i j}\left(\frac{x}{c}\right) b_{i j}(x+y)\right\} d x \\
& +\sum_{i=1}^{r} \sum_{j=1}^{m_{i}} \sum_{l=0}^{n}\left(\begin{array}{c}
n \\
l
\end{array}\right) \int_{0}^{\infty} \int_{0}^{x} \int_{x / c}^{\infty} \int_{0}^{\infty} e^{-\delta_{l}^{*} t} z^{n-l} f^{l}(x+y) g_{\delta_{2}}^{*}(t, z, x, v \mid 0) \frac{\frac{1}{c} k_{i j}\left(\frac{x-v}{c}\right) b_{i j}(x+y)}{\int_{0}^{\infty} g_{1}(x, s \mid v) d s} d z d t d v d x,
\end{aligned}
$$

where a change of variable for the integral involving $z$ and a binomial expansion have been performed. From the above equation, it is clear that the dependence of $h_{\delta_{1}, \delta_{2}, n}(y \mid 0)$ on $y$ only appears through $b_{i j}(\cdot)$ 's and the cost function $f(\cdot)$. Further assumptions on these quantities need to be made.

In the rest of this section, for simplicity it is assumed that $m_{i}=1$, and we denote $k_{i}(\cdot)=k_{i 1}(\cdot)$, $\widetilde{k}_{i}(\cdot)=\widetilde{k}_{i 1}(\cdot)$ and $b_{i}(\cdot)=b_{i 1}(\cdot)$ for $i=1,2, \ldots, r$. In particular, we assume that $b_{i}(y)=\mu_{i} e^{-\mu_{i} y}$ is an exponential density. Hence, (1.1) becomes

$$
p(t, y)=\sum_{i=1}^{r} k_{i}(t) \mu_{i} e^{-\mu_{i} y}, \quad t, y>0 .
$$

Here $k_{i}(\cdot)$ 's are not necessarily densities, and $\mu_{i}$ 's are assumed distinct. Under (4.3), the marginal distribution of the claim size $Y$ follows a combination of exponentials. This reverse type of dependency structure (4.3) was studied by Landriault et al. (2013), who analyzed the Gerber-Shiu function in which the penalty function depends on the deficit only. Since we are mostly interested in the discounted aggregate claims until ruin, it is assumed that $w(x, y, v) \equiv 1$ and $f(x)=x$. Therefore, the Gerber-Shiu 
function (1.3) becomes

$$
\phi_{\delta_{12}, n}(u)=E\left[e^{-\delta_{1} \tau}\left(\sum_{i=1}^{N(\tau)} e^{-\delta T_{i}} Y_{i}\right)^{n} I\{\tau<\infty\} \mid U(0)=u\right], \quad u \geq 0 .
$$

It is remarked we do not assume $\delta_{1}=0$ here so as to facilitate the study of covariance of $\tau$ and $\sum_{i=1}^{N(\tau)} e^{-\delta T_{i}} Y_{i}$ in Section 5. For later use, for each fixed $n=0,1, \ldots$, we define the Lundberg's equation (in $\xi$ )

$$
\widetilde{p}\left(\delta_{n}^{*}-c \xi, \xi\right)=\sum_{i=1}^{r} \widetilde{k}_{i}\left(\delta_{n}^{*}-c \xi\right) \frac{\mu_{i}}{\mu_{i}+\xi}=1,
$$

which is known to have exactly $r$ roots with negative real parts (see Landriault et al. (2013)).

Under the above assumptions, by applying a binomial expansion to the term $f^{l}(x+y)=(x+y)^{l}$ in (4.2), it is not difficult to see that $h_{\delta_{1}, \delta_{2}, n}(y \mid 0)$ admits the form

$$
h_{\delta_{1}, \delta_{2}, n}(y \mid 0)=\sum_{j=0}^{n} \sum_{k=1}^{r} C_{\delta_{1}, \delta_{2}, j, k, n} \frac{y^{j} e^{-\mu_{k} y}}{j !}, \quad y>0
$$

for some constants $C_{\delta_{1}, \delta_{2}, j, k, n}$ 's. The corresponding Laplace transform $\widetilde{h}_{\delta_{1}, \delta_{2}, n}(s \mid 0)=\int_{0}^{\infty} e^{-s y} h_{\delta_{1}, \delta_{2}, n}(y \mid 0) d y$ is thus given by

$$
\widetilde{h}_{\delta_{1}, \delta_{2}, n}(s \mid 0)=\sum_{j=0}^{n} \sum_{k=1}^{r} \frac{C_{\delta_{1}, \delta_{2}, j, k, n}}{\left(\mu_{k}+s\right)^{j+1}}
$$

Now, (4.6) will in turn be used to identify the appropriate solution form of $\phi_{\delta_{1}, \delta_{2}, n}(u)$ as a function of $u$. Using (2.6), (2.11) reduces to

$\phi_{\delta_{12}, n}(u)=\int_{0}^{u} h_{\delta_{n}^{*}, \delta_{2}, 0}(y \mid 0) \phi_{\delta_{12}, n}(u-y) d y+\sum_{j=0}^{n-1}\left(\begin{array}{c}n \\ j\end{array}\right) \int_{0}^{u} h_{\delta_{j}^{*}, \delta_{2}, n-j}(y \mid 0) \phi_{\delta_{12}, j}(u-y) d y+\int_{u}^{\infty} h_{\delta_{1}, \delta_{2}, n}(y \mid 0) d y$

With the help of (4.6), it can be readily verified that

$$
\int_{0}^{\infty} e^{-s u} \int_{u}^{\infty} h_{\delta_{1}, \delta_{2}, n}(y \mid 0) d y d u=\sum_{j=0}^{n} \sum_{k=1}^{r} \frac{C_{\delta_{1}, \delta_{2}, j, k, n}^{*}}{\left(\mu_{k}+s\right)^{j+1}}
$$

for some constants $C_{\delta_{1}, \delta_{2}, j, k, n}^{*}$ 's. Hence, taking Laplace transforms on both sides of (4.8) followed by rearrangements yields

$$
\widetilde{\phi}_{\delta_{12}, n}(s)=\frac{1}{1-\widetilde{h}_{\delta_{n}^{*}, \delta_{2}, 0}(s \mid 0)}\left\{\sum_{j=0}^{n-1}\left(\begin{array}{c}
n \\
j
\end{array}\right) \widetilde{h}_{\delta_{j}^{*}, \delta_{2}, n-j}(s \mid 0) \widetilde{\phi}_{\delta_{12}, j}(s)+\sum_{j=0}^{n} \sum_{k=1}^{r} \frac{C_{\delta_{1}, \delta_{2}, j, k, n}^{*}}{\left(\mu_{k}+s\right)^{j+1}}\right\} .
$$

For each fixed $n=0,1, \ldots$, define $\left\{-\kappa_{k, n}\right\}_{k=1}^{r}$ to be the roots of the polynomial equation (in $\xi$ )

$$
\prod_{k=1}^{r}\left(\mu_{k}+\xi\right)-\sum_{k=1}^{r} C_{\delta_{n}^{*}, \delta_{2}, 0, k, 0} \prod_{i=1, i \neq k}^{r}\left(\mu_{i}+\xi\right)=0
$$


These roots are known to have negative real parts and are assumed to be distinct (see Remark 1). Then, by applying (4.7) to (4.9), we arrive at

$$
\begin{aligned}
\widetilde{\phi}_{\delta_{12}, n}(s)=\frac{1}{\prod_{k=1}^{r}\left(s+\kappa_{k, n}\right)}\{ & \sum_{j=0}^{n-1}\left(\begin{array}{c}
n \\
j
\end{array}\right) \sum_{l=0}^{n-j} \sum_{k=1}^{r} \frac{C_{\delta_{j}^{*}, \delta_{2}, l, k, n-j} \prod_{i=1, i \neq k}^{r}\left(\mu_{i}+s\right)}{\left(\mu_{k}+s\right)^{l}} \widetilde{\phi}_{\delta_{12}, j}(s) \\
& \left.+\sum_{j=0}^{n} \sum_{k=1}^{r} \frac{C_{\delta_{1}, \delta_{2}, j, k, n}^{*} \prod_{i=1, i \neq k}^{r}\left(\mu_{i}+s\right)}{\left(\mu_{k}+s\right)^{j}}\right\} .
\end{aligned}
$$

By induction, it can be deduced that $\widetilde{\phi}_{\delta_{12}, n}(s)$ admits the partial fractions representation

$$
\widetilde{\phi}_{\delta_{12}, n}(s)=\sum_{j=0}^{n-1} \sum_{k=1}^{r} \frac{B_{j, k, n}}{\left(\mu_{k}+s\right)^{j+1}}+\sum_{j=0}^{n} \sum_{k=1}^{r} \frac{B_{j, k, n}^{*}}{s+\kappa_{k, j}},
$$

for some constants $B_{j, k, n}$ 's and $B_{j, k, n}^{*}$ 's. Thus, Laplace transform inversion gives

$$
\phi_{\delta_{12}, n}(u)=\sum_{j=0}^{n-1} \sum_{k=1}^{r} B_{j, k, n} \frac{u^{j} e^{-\mu_{k} u}}{j !}+\sum_{j=0}^{n} \sum_{k=1}^{r} B_{j, k, n}^{*} e^{-\kappa_{k, j} u}, \quad u \geq 0 .
$$

Having identified the above solution form, the last step is to determine the unknown coefficients by back substitution into the integral equation (2.20). From Cheung (2013, Section 3.1), it has been shown that the recursive evaluation of these coefficients is extremely tedious for general positive integer $n$ even for the simple case of the traditional Sparre Andersen model with exponential claims. Therefore, for illustration we only focus on $\phi_{\delta_{12}, 1}(u)$ which will result in a neat expression. In particular, it will be shown that $B_{0, k, 1}=0$ for $k=1,2, \ldots, r$, i.e. the first summation in (4.10) vanishes. When $n=1$, it is clear from (2.20) that one requires the starting point $\phi_{\delta_{12}, 0}(\cdot)$ via $\phi_{\delta_{12}, 1}^{*}(x, y)$ in $(2.23)$. From $(4.4), \phi_{\delta_{12}, 0}(\cdot)$ is simply the Laplace transform of the time of ruin. Hence, according to Landriault et al. (2013, Theorem 1), one asserts that (4.10) holds true, where $\left\{-\kappa_{k, 0}\right\}_{k=1}^{r}$ are the $r$ roots with negative real parts to (4.5) under $n=0$, and $\left\{B_{0, k, 0}^{*}\right\}_{k=1}^{r}$ can be obtained from the system of linear equations

$$
\sum_{k=1}^{r} B_{0, k, 0}^{*} \frac{\mu_{i}}{\mu_{i}-\kappa_{k, 0}}=1, \quad i=1,2, \ldots, r .
$$

When $w(x, y, v) \equiv 1$ and $f(x)=x$, the use of (2.21) and (2.23) leads (2.20) for $n=1$ to

$$
\begin{aligned}
\phi_{\delta_{12}, 1}(u)=\sum_{i=1}^{r} \int_{0}^{\infty} e^{-\left(\delta_{1}+\delta_{2}\right) t}\{ & \int_{u+c t}^{\infty} y \mu_{i} e^{-\mu_{i} y} d y+\int_{0}^{u+c t} \phi_{\delta_{12}, 1}(u+c t-y) \mu_{i} e^{-\mu_{i} y} d y \\
& \left.+\int_{0}^{u+c t} y \phi_{\delta_{12}, 0}(u+c t-y) \mu_{i} e^{-\mu_{i} y} d y\right\} k_{i}(t) d t .
\end{aligned}
$$

We evaluate three double integrals on the right-hand side of the above equation as follows. The first and third ones are rather straightforward, and we omit the algebra and simply state that

$$
\begin{aligned}
& \sum_{i=1}^{r} \int_{0}^{\infty} e^{-\left(\delta_{1}+\delta_{2}\right) t}\left\{\int_{u+c t}^{\infty} y \mu_{i} e^{-\mu_{i} y} d y\right\} k_{i}(t) d t \\
= & \sum_{i=1}^{r} \widetilde{k}_{i}\left(\delta_{1}+\delta_{2}+c \mu_{i}\right) u e^{-\mu_{i} u}+\sum_{i=1}^{r}\left\{\frac{1}{\mu_{i}} \widetilde{k}_{i}\left(\delta_{1}+\delta_{2}+c \mu_{i}\right)+c \mathcal{T}_{\delta_{1}+\delta_{2}+c \mu_{i}}^{2} k_{i}(0)\right\} e^{-\mu_{i} u},
\end{aligned}
$$


and

$$
\begin{aligned}
& \sum_{i=1}^{r} \int_{0}^{\infty} e^{-\left(\delta_{1}+\delta_{2}\right) t}\left\{\int_{0}^{u+c t} y \phi_{\delta_{12}, 0}(u+c t-y) \mu_{i} e^{-\mu_{i} y} d y\right\} k_{i}(t) d t \\
= & \sum_{k=1}^{r} B_{0, k, 0}^{*} \sum_{i=1}^{r} \frac{\mu_{i}}{\left(\mu_{i}-\kappa_{k, 0}\right)^{2}} \widetilde{k}_{i}\left(\delta_{1}+\delta_{2}+c \kappa_{k, 0}\right) e^{-\kappa_{k, 0} u}-\sum_{i=1}^{r} \widetilde{k}_{i}\left(\delta_{1}+\delta_{2}+c \mu_{i}\right) \sum_{k=1}^{r} B_{0, k, 0}^{*} \frac{\mu_{i}}{\mu_{i}-\kappa_{k, 0}} u e^{-\mu_{i} u} \\
& -\sum_{i=1}^{r} \sum_{k=1}^{r} B_{0, k, 0}^{*} \frac{\mu_{i}}{\mu_{i}-\kappa_{k, 0}}\left\{\frac{1}{\mu_{i}-\kappa_{k, 0}} \widetilde{k}_{i}\left(\delta_{1}+\delta_{2}+c \mu_{i}\right)+c \mathcal{T}_{\delta_{1}+\delta_{2}+c \mu_{i}}^{2} k_{i}(0)\right\} e^{-\mu_{i} u},
\end{aligned}
$$

where $\mathcal{T}_{\delta_{1}+\delta_{2}+\mu_{i} c}^{2} k_{i}(0)=\int_{0}^{\infty} t e^{-\left(\delta_{1}+\delta_{2}+\mu_{i} c\right) t} k_{i}(t) d t$ corresponds to the double Dickson-Hipp operator (see Dickson and Hipp (2001) and Li and Garrido (2004)). For the second double integral in (4.12), one needs to be careful in computing the inner integral $\int_{0}^{u+c t} \phi_{\delta_{12}, 1}(u+c t-y) \mu_{i} e^{-\mu_{i} y} d y$, as $\phi_{\delta_{12}, 1}(u+c t-y)$ involves exponential terms in the form of $e^{-\mu_{k}(u+c t-y)}$ which has some cancellations with $e^{-\mu_{i} y}$ when $i=k$. After all, it is found that

$$
\begin{aligned}
& \sum_{i=1}^{r} \int_{0}^{\infty} e^{-\left(\delta_{1}+\delta_{2}\right) t}\left\{\int_{0}^{u+c t} \phi_{\delta_{12}, 1}(u+c t-y) \mu_{i} e^{-\mu_{i} y} d y\right\} k_{i}(t) d t \\
= & \sum_{i=1}^{r} \widetilde{k}_{i}\left(\delta_{1}+\delta_{2}+c \mu_{i}\right) B_{0, i, 1} \mu_{i} u e^{-\mu_{i} u}+\sum_{i=1}^{r} B_{0, i, 1} c \mu_{i} \mathcal{T}_{\delta_{1}+\delta_{2}+c \mu_{i}}^{2} k_{i}(0) e^{-\mu_{i} u} \\
& +\sum_{i=1}^{r} \sum_{k=1, k \neq i}^{r} B_{0, k, 1} \frac{\mu_{i}}{\mu_{i}-\mu_{k}}\left\{\widetilde{k}_{i}\left(\delta_{1}+\delta_{2}+c \mu_{k}\right) e^{-\mu_{k} u}-\widetilde{k}_{i}\left(\delta_{1}+\delta_{2}+c \mu_{i}\right) e^{-\mu_{i} u}\right\} \\
& +\sum_{j=0}^{1} \sum_{k=1}^{r} B_{j, k, 1}^{*} \sum_{i=1}^{r} \frac{\mu_{i}}{\mu_{i}-\kappa_{k, j}} \widetilde{k}_{i}\left(\delta_{1}+\delta_{2}+c \kappa_{k, j}\right) e^{-\kappa_{k, j} u}-\sum_{i=1}^{r} \widetilde{k}_{i}\left(\delta_{1}+\delta_{2}+c \mu_{i}\right) \sum_{j=0}^{1} \sum_{k=1}^{r} B_{j, k, 1}^{*} \frac{\mu_{i}}{\mu_{i}-\kappa_{k, j}} e^{-\mu_{i} u} .
\end{aligned}
$$

We proceed by substituting (4.13)-(4.15) into (4.12) and equating the coefficients of various exponential terms with (4.10) for $n=1$. First, the coefficients of $e^{-\kappa_{k, 1} u}(k=1,2, \ldots, r)$ imply that $\left\{-\kappa_{k, 1}\right\}_{k=1}^{r}$ are the $r$ roots to (4.5) (when $n=1$ ) with negative real parts. Similarly, equating the coefficients of $e^{-\kappa_{k, 0} u}$ and rearranging terms, we arrive at

$$
B_{0, k, 1}^{*}=\frac{B_{0, k, 0}^{*} \sum_{i=1}^{r} \frac{\mu_{i}}{\left(\mu_{i}-\kappa_{k, 0}\right)^{2}} \widetilde{k}_{i}\left(\delta_{1}+\delta_{2}+c \kappa_{k, 0}\right)}{1-\sum_{i=1}^{r} \frac{\mu_{i}}{\mu_{i}-\kappa_{k, 0}} \widetilde{k}_{i}\left(\delta_{1}+\delta_{2}+c \kappa_{k, 0}\right)}, \quad k=1,2, \ldots, r .
$$

In the above equation, $B_{0, k, 1}^{*}$ is expressed in terms of $B_{0, k, 0}^{*}$ which is known because of (4.11). Next, the coefficients of $u e^{-\mu_{i} u}$ together with the fact that $B_{0, k, 0}^{*}$ 's satisfy (4.11) lead to

$$
B_{0, i, 1}=0, \quad i=1,2, \ldots, r .
$$

Finally, we equate the coefficients of $e^{-\mu_{i} u}$ and apply (4.17) to arrive at

$$
\begin{aligned}
0= & \frac{1}{\mu_{i}} \widetilde{k}_{i}\left(\delta_{1}+\delta_{2}+c \mu_{i}\right)+c \mathcal{T}_{\delta_{1}+\delta_{2}+c \mu_{i}}^{2} k_{i}(0)-\widetilde{k}_{i}\left(\delta_{1}+\delta_{2}+c \mu_{i}\right) \sum_{j=0}^{1} \sum_{k=1}^{r} B_{j, k, 1}^{*} \frac{\mu_{i}}{\mu_{i}-\kappa_{k, j}} \\
& -\sum_{k=1}^{r} B_{0, k, 0}^{*} \frac{\mu_{i}}{\mu_{i}-\kappa_{k, 0}}\left\{\frac{1}{\mu_{i}-\kappa_{k, 0}} \widetilde{k}_{i}\left(\delta_{1}+\delta_{2}+c \mu_{i}\right)+c \mathcal{T}_{\delta_{1}+\delta_{2}+c \mu_{i}}^{2} k_{i}(0)\right\}, \quad i=1,2, \ldots, r .
\end{aligned}
$$


The only unknowns in the above equation are $\left\{B_{1, k, 1}^{*}\right\}_{k=1}^{r}$, since $\left\{B_{0, k, 0}^{*}\right\}_{k=1}^{r}$ can be solved from (4.11) and then $\left\{B_{0, k, 1}^{*}\right\}_{k=1}^{r}$ are explicitly given by (4.16). Hence, (4.18) forms a linear system of $r$ equations from which $\left\{B_{1, k, 1}^{*}\right\}_{k=1}^{r}$ can be solved for. To summarize, because of (4.17), (4.10) for $n=1$ simplifies to give

$$
\phi_{\delta_{12}, 1}(u)=\sum_{j=0}^{1} \sum_{k=1}^{r} B_{j, k, 1}^{*} e^{-\kappa_{k, j} u}, \quad u \geq 0 .
$$

\section{$5 \quad$ Numerical illustrations}

In this section, we apply the results in preceding sections to find, conditional on ruin occurring, (i) the covariance of the time of ruin $\tau$ and the discounted aggregate claims until ruin $\sum_{i=1}^{N(\tau)} e^{-\delta_{2} T_{i}} Y_{i}$; and (ii) the expectation, variance and third central moment of $\sum_{i=1}^{N(\tau)} e^{-\delta_{2} T_{i}} Y_{i}$. Throughout it is sufficient to use the cost function $f(x)=x$ so that $Z_{\delta_{2}}(\tau)=\sum_{i=1}^{N(\tau)} e^{-\delta_{2} T_{i}} Y_{i}$. For notational convenience, we shall use $E_{u}$ to denote the expectation taken under $U(0)=u$.

First, we introduce the conditional covariance defined by

$$
\begin{aligned}
\operatorname{Cov}\left(\tau, Z_{\delta_{2}}(\tau) \mid u\right) & =E_{u}\left[\tau Z_{\delta_{2}}(\tau) \mid \tau<\infty\right]-E_{u}[\tau \mid \tau<\infty] E_{u}\left[Z_{\delta_{2}}(\tau) \mid \tau<\infty\right] \\
& =\frac{E_{u}\left[\tau Z_{\delta_{2}}(\tau) I\{\tau<\infty\}\right]}{\psi(u)}-\frac{E_{u}[\tau I\{\tau<\infty\}] E_{u}\left[Z_{\delta_{2}}(\tau) I\{\tau<\infty\}\right]}{\psi^{2}(u)} .
\end{aligned}
$$

(Interested readers are referred to Psarrakos and Politis (2012) for the study of the covariance of the surplus prior to ruin and the deficit at ruin in the classical compound Poisson risk model.) In addition, we are also interested in the following three moment-based quantities in relation to $Z_{\delta_{2}}(\tau)$ conditional on ruin, namely the mean

$$
\operatorname{Mean}\left(Z_{\delta_{2}}(\tau) \mid u\right)=E_{u}\left[Z_{\delta_{2}}(\tau) \mid \tau<\infty\right]=\frac{E_{u}\left[Z_{\delta_{2}}(\tau) I\{\tau<\infty\}\right]}{\psi(u)},
$$

the variance

$\operatorname{Var}\left(Z_{\delta_{2}}(\tau) \mid u\right)=E_{u}\left[\left\{Z_{\delta_{2}}(\tau)-\operatorname{Mean}\left(Z_{\delta_{2}}(\tau) \mid u\right)\right\}^{2} \mid \tau<\infty\right]=\frac{E_{u}\left[Z_{\delta_{2}}^{2}(\tau) I\{\tau<\infty\}\right]}{\psi(u)}-\left(\frac{E_{u}\left[Z_{\delta_{2}}(\tau) I\{\tau<\infty\}\right]}{\psi(u)}\right)^{2}$,

and the third central moment

$$
\begin{aligned}
\mathrm{CM}_{3}\left(Z_{\delta_{2}}(\tau) \mid u\right) & =E_{u}\left[\left\{Z_{\delta_{2}}(\tau)-\operatorname{Mean}\left(Z_{\delta_{2}}(\tau) \mid u\right)\right\}^{3} \mid \tau<\infty\right] \\
& =\frac{E_{u}\left[Z_{\delta_{2}}^{3}(\tau) I\{\tau<\infty\}\right]}{\psi(u)}-3 \times \operatorname{Mean}\left(Z_{\delta_{2}}(\tau) \mid u\right) \times \operatorname{Var}\left(Z_{\delta_{2}}(\tau) \mid u\right)-\operatorname{Mean}^{3}\left(Z_{\delta_{2}}(\tau) \mid u\right) .
\end{aligned}
$$

The evaluation of various quantities involved in the above definitions is explained as follows.

- $\psi(u)$ is the ruin probability (i.e. a special case of $\phi_{\delta_{12}, 0}(u)$ in $(1.3)$ when $w(x, y, v) \equiv 1$ and $\left.\delta_{1}=0\right)$.

- One can find

$$
E_{u}\left[\tau Z_{\delta_{2}}(\tau) I\{\tau<\infty\}\right]=-\left.\frac{\partial}{\partial \delta_{1}} E_{u}\left[e^{-\delta_{1} \tau} Z_{\delta_{2}}(\tau) I\{\tau<\infty\}\right]\right|_{\delta_{1}=0}, \quad u \geq 0
$$


where the expectation on the right-hand side is a special case of $\phi_{\delta_{12}, 1}(u)$ in $(1.3)$ with $w(x, y, v) \equiv 1$ and $f(x)=x$. In particular, if the dependency (4.3) holds, then it can be seen from (4.19) that the above quantity (as a function of $u$ ) is a linear sum of terms involving $e^{-\kappa_{k, j} u}$ 's and $u e^{-\kappa_{k, j} u}$ 's for $j=0,1$ and $k=1,2, \ldots, r$. (Note that $B_{j, k, 1}^{*}$ 's and $-\kappa_{k, j}$ 's in (4.19) depend on $\delta_{1}$ implicitly.)

- Similarly, the expected time to ruin $E_{u}[\tau I\{\tau<\infty\}]$ can be obtained by differentiating $\phi_{\delta_{12}, 0}(u)$ with $w(x, y, v) \equiv 1$.

- The $n$-th moment $E_{u}\left[Z_{\delta_{2}}^{n}(\tau) I\{\tau<\infty\}\right]$ is a special case of $\phi_{\delta_{12}, n}(u)$ with $w(x, y, v) \equiv 1 ; f(x)=x$ and $\delta_{1}=0$.

For numerical illustrations, it is assumed that the interclaim time $V$ and the claim severity $Y$ are both exponentially distributed with means $1 / \lambda$ and $1 / \mu$ respectively. In addition, the dependency between $V$ and $Y$ is modelled via the Farlie-Gumbel-Morgenstern (FGM) copula (e.g. Nelsen (2006, Examples 3.12 and 5.2)). We refer interested readers to e.g. Cossette et al. (2010) and Chadjiconstantinidis and Vrontos (2012) for the study of risk processes under FGM copula. Therefore, the joint density of $(V, Y)$ is given by

$$
p(t, y)=\left(\lambda e^{-\lambda t}\right)\left(\mu e^{-\mu y}\right)+\theta\left(\lambda e^{-\lambda t}\right)\left(\mu e^{-\mu y}\right)\left(2 e^{-\lambda t}-1\right)\left(2 e^{-\mu y}-1\right), \quad y, t>0 .
$$

Here $\theta$ is the dependency parameter such that $-1 \leq \theta \leq 1$, with $\theta=0$ corresponding to the case of independence. Clearly, the above joint density can be put in the form of (1.1) under (3.1) or (4.3), and therefore both Sections 3 and 4 are applicable. We shall closely follow the parameters in Cossette et al. (2010, Example 8.1), and let $\lambda=\mu=1$ and $c=1.5$.

We first plot the conditional covariance $\operatorname{Cov}\left(\tau, Z_{\delta_{2}}(\tau) \mid u\right)$ against the initial surplus $u$ for the dependency parameters $\theta=-1,-0.5,0,0.5,1$ when $\delta_{2}=0.01$ in Figure 1 and when $\delta_{2}=0.05$ in Figure 2. From both figures, for each fixed $\theta$, as $u$ increases the covariance always first increases starting from a positive value, and then decreases to negative values and finally converges. The sign change of the covariance as the initial surplus increases may be interpreted as follows. In general, conditional on ruin, the aggregate claims until ruin (without discounting), namely $\sum_{i=1}^{N(\tau)} Y_{i}$, is always given by $u+c \tau+|U(\tau)|$. For a fixed initial surplus $u$, there are two opposing effects to the discounted aggregate claims $Z_{\delta_{2}}(\tau)=\sum_{i=1}^{N(\tau)} e^{-\delta_{2} T_{i}} Y_{i}$ if $\tau$ gets larger: (i) the aggregate claims $\sum_{i=1}^{N(\tau)} Y_{i}$ (and hence $\sum_{i=1}^{N(\tau)} e^{-\delta_{2} T_{i}} Y_{i}$ ) tends to be larger; but (ii) the occurrences of these claims are spread over a longer time period as the process survives longer, and the effect of discounting tends to make $\sum_{i=1}^{N(\tau)} e^{-\delta_{2} T_{i}} Y_{i}$ smaller. When $u$ is small, the numerical result of positive covariance indicates that the former effect dominates. However, as $u$ increases further, the sign change of the covariance suggests that the latter effect becomes more dominant because the discounting on $u$ gets more significant. In addition to the pattern of $\operatorname{Cov}\left(\tau, Z_{\delta_{2}}(\tau) \mid u\right)$ as a function of $u$, we note that for almost all values of fixed $u$ the absolute value of the covariance decreases when the dependency parameter $\theta$ increases from -1 to 1 . Finally, the absolute value of the covariance is mostly smaller in Figure 2 (i.e. $\delta_{2}=0.05$ ) than in Figure 1 (i.e. $\delta_{2}=0.01$ ), which can be attributed to the fact that the discounted aggregate claims $Z_{\delta_{2}}(\tau)$ is always decreasing in $\delta_{2}$.

\section{INSERT FIGURE 1}

Figure 1 : Plot of $\operatorname{Cov}\left(\tau, Z_{0.01}(\tau) \mid u\right)$ for various choices of $\theta$ 


\section{INSERT FIGURE 2}

Figure 2 : Plot of $\operatorname{Cov}\left(\tau, Z_{0.05}(\tau) \mid u\right)$ for various choices of $\theta$

Next, the moment-based quantities $\operatorname{Mean}\left(Z_{\delta_{2}}(\tau) \mid u\right), \operatorname{Var}\left(Z_{\delta_{2}}(\tau) \mid u\right)$ and $\mathrm{CM}_{3}\left(Z_{\delta_{2}}(\tau) \mid u\right)$ for $\delta_{2}=0.01$ and $\delta_{2}=0.05$ are plotted in Figures 3-8. Analogous to the comparison across Figures 1 and 2, the values in Figures 3,5 and 7 (i.e. $\delta_{2}=0.01$ ) are mostly larger than those in Figures 4,6 and 8 (i.e. $\delta_{2}=0.05$ ) because the magnitude of $Z_{\delta_{2}}(\tau)$ is larger when $\delta_{2}$ is smaller. In both Figures 3 and 4 , the conditional mean Mean $\left(Z_{\delta_{2}}(\tau) \mid u\right)$ is an increasing function of $u$ for fixed dependency parameter $\theta$. This makes sense as more claims are required to cause ruin if one starts with larger initial surplus. However, within each of these two figures, the curves for different values of $\theta$ are very close to each other, which suggests that $\operatorname{Mean}\left(Z_{\delta_{2}}(\tau) \mid u\right)$ is fairly insensitive to the level of dependency between an interclaim time and the resulting claim amount. When we turn to the conditional variance $\operatorname{Var}\left(Z_{\delta_{2}}(\tau) \mid u\right)$, Figures 5 and 6 show the similar pattern: $\operatorname{Var}\left(Z_{\delta_{2}}(\tau) \mid u\right)$ is not monotone in $u$ but it converges to a finite value as $u \rightarrow \infty$. In addition, $\operatorname{Var}\left(Z_{\delta_{2}}(\tau) \mid u\right)$ increases when $\theta$ decreases. Interestingly, Bargès et al. (2011, Section 4.1) and Woo and Cheung (2013, Section 4.1) also illustrated the same phenomenon for the discounted aggregate claims

until a fixed time $t$, namely $Z_{\delta_{2}}(t)=\sum_{i=1}^{N(t)} e^{-\delta_{2} T_{i}} Y_{i}$. The same probabilistic interpretations therein are applicable: if an interclaim time and the resulting claim amount are negatively dependent, a large (small) claim is usually associated to a small (large) interclaim time and hence the discounted value each claim amount is more likely to take on extreme values, leading to a larger variance to $\operatorname{Var}\left(Z_{\delta_{2}}(\tau) \mid u\right)$. Finally, from Figures 7 and 8, it can be seen that the third central moment $\mathrm{CM}_{3}\left(Z_{\delta_{2}}(\tau) \mid u\right)$ is not monotone in $u$. In particular, $\mathrm{CM}_{3}\left(Z_{\delta_{2}}(\tau) \mid u\right)$ mostly takes on positive values, i.e. $Z_{\delta_{2}}(\tau)$ is skewed to the right except for a small domain of $u$. Nonetheless, similar to the case of $\operatorname{Cov}\left(\tau, Z_{\delta_{2}}(\tau) \mid u\right)$, for most values of fixed $u$ the absolute value of $\mathrm{CM}_{3}\left(Z_{\delta_{2}}(\tau) \mid u\right)$ decreases when the dependency parameter $\theta$ increases.

\section{INSERT FIGURE 3}

Figure 3 : Plot of Mean $\left(Z_{0.01}(\tau) \mid u\right)$ for various choices of $\theta$

\section{INSERT FIGURE 4}

Figure 4: Plot of Mean $\left(Z_{0.05}(\tau) \mid u\right)$ for various choices of $\theta$

\section{INSERT FIGURE 5}

Figure 5 : Plot of $\operatorname{Var}\left(Z_{0.01}(\tau) \mid u\right)$ for various choices of $\theta$

\section{INSERT FIGURE 6}

Figure 6 : Plot of $\operatorname{Var}\left(Z_{0.05}(\tau) \mid u\right)$ for various choices of $\theta$ 


\title{
INSERT FIGURE 7
}

Figure $7:$ Plot of $\mathrm{CM}_{3}\left(Z_{0.01}(\tau) \mid u\right)$ for various choices of $\theta$

\author{
INSERT FIGURE 8
}

Figure 8 : Plot of $\mathrm{CM}_{3}\left(Z_{0.05}(\tau) \mid u\right)$ for various choices of $\theta$

\section{Acknowledgements}

The authors would like to thank the anonymous referee and the editor for helpful comments and suggestions which improved an earlier version of the paper. Eric Cheung gratefully acknowledges the support from the Research Grants Council of the Hong Kong Special Administrative Region (Project Number: HKU 701212P). The research of J.-K. Woo is supported by the Seed Funding for Basic Research (Project Number: 201302159004) provided by the University of Hong Kong as well as the CAE 2013 research grant from the Society of Actuaries. Any opinions, finding, and conclusions or recommendations expressed in this material are those of the authors and do not necessarily reflect the views of the SOA.

\section{References}

[1] Albrecher, H. And Teugels, J.L. 2006. Exponential behavior in the presence of dependence in risk theory. Journal of Applied Probability 43(1): 257-273.

[2] Ambagaspitiya, R.S. 2009. Ultimate ruin probability in the Sparre Andersen model with dependent claim sizes and claim occurrence times. Insurance: Mathematics and Economics 44(3): 464-472.

[3] Asmussen, S. 2003. Applied Probability and Queues. 2nd Edition. New York: Springer.

[4] Badescu, A.L., Cheung, E.C.K. And Landriault, D. 2009. Dependent risk models with bivariate phase-type distributions. Journal of Applied Probability 46(1): 113-131.

[5] Bargès, M., Cossette, H., Loisel, S. and Marceau, È. 2011. On the moments of aggregate discounted claims with dependence introduced by a FGM copula. ASTIN Bulletin 41(1): 215-238.

[6] Boudreault, M., Cossette, H., Landriault, D. and Marceau, E. 2006. On a risk model with dependence between interclaim arrivals and claim sizes. Scandinavian Actuarial Journal 2006(5): 265-285.

[7] Cai, J., Feng, R. And Willmot, G.E. 2009. On the total discounted operating costs up to default and its applications. Advances in Applied Probability 41(2): 495-522.

[8] Chadjiconstantinidis, S. and Vrontos, S. 2012. On a renewal risk process with dependence under a Farlie-Gumbel-Morgenstern copula. Scandinavian Actuarial Journal. In press.

[9] Cheung, E.C.K. 2011. A generalized penalty function in Sparre Andersen risk models with surplusdependent premium. Insurance: Mathematics and Economics 48(3): 384-397. 
[10] Cheung, E.C.K. 2013. Moments of discounted aggregate claim costs until ruin in a Sparre Andersen risk model with general interclaim times. Insurance: Mathematics and Economics 53(2): 343-354.

[11] Cheung, E.C.K. And Feng, R. 2013. A unified analysis of claim costs up to ruin in a Markovian arrival risk process. Insurance: Mathematics and Economics 53(1): 98-109.

[12] Cheung, E.C.K., Landriault, D., Willmot, G.E. And Woo, J.-K. 2010a. Gerber-Shiu analysis with a generalized penalty function. Scandinavian Actuarial Journal 2010(3): 185-199.

[13] Cheung, E.C.K., Landriault, D., Willmot, G.E. and Woo, J.-K. 2010b. Structural properties of Gerber-Shiu functions in dependent Sparre Andersen models. Insurance: Mathematics and Economics 46(1): 117-126.

[14] Cheung, E.C.K., Landriault, D., Willmot, G.E. And Woo, J.-K. 2011. On orderings and bounds in a generalized Sparre Andersen risk model. Applied Stochastic Models in Business and Industry 27(1): 51-60.

[15] Cossette, H., Marceau, E. And Marri, F. 2008. On the compound Poisson risk model with dependence based on a generalized Farlie-Gumbel-Morgenstern copula. Insurance: Mathematics and Economics 43(3): 444-455.

[16] Cossette, H., Marceau, E. And Marri, F. 2010. Analysis of ruin measures for the classical compound Poisson risk model with dependence. Scandinavian Actuarial Journal 2010(3): 221-245.

[17] D'Auria, B., Ivanovs, J., Kella, O. and Mandjes, M. 2010. First passage of a Markov additive process and generalized Jordan chains. Journal of Applied Probability 47(4): 1048-1057.

[18] Dickson, D.C.M. And Hipp, C. 2001. On the time to ruin for Erlang(2) risk processes. Insurance: Mathematics and Economics 29(3): 333-344.

[19] Feng, R. 2009a. On the total operating costs up to default in a renewal risk model. Insurance: Mathematics and Economics 45(2): 305-314.

[20] Feng, R. 2009b. A matrix operator approach to the analysis of ruin-related quantities in the phasetype renewal risk model. Bulletin of the Swiss Association of Actuaries 2009(1\&2): 71-87.

[21] Gerber, H.U. And Shiu, E.S.W. 1998. On the time value of ruin. North American Actuarial Journal 2(1): 48-72.

[22] Ji, L, AND Zhang, C. 2012. Analysis of the multiple roots of the Lundberg fundamental equation in the $\mathrm{PH}(n)$ risk model. Applied Stochastic Models in Business and Industry 28(1): 73-90.

[23] Labbé, C., Sendov, H.S. And Sendova, K.P. 2011. The Gerber-Shiu function and the generalized Cramér-Lundberg model. Applied Mathematics and Computation 218(7): 3035-3056.

[24] Landriault, D., Lee, W.Y., Willmot, G.E. And Woo, J.-K. 2013. A note on deficit analysis in dependency models involving Coxian claim amounts. Scandinavian Actuarial Journal. In press.

[25] Li, S. AND Garrido, J. 2004. On ruin for the $\operatorname{Erlang}(n)$ risk process. Insurance: Mathematics and Economics 34(3): 391-408.

[26] Lindsay, B.G., Pilla, R.S. And Basak, P. 2000. Moment-based approximations of distributions using mixtures: Theory and applications. Annals of the Institute of Statistical Mathematics 52(2): 215-230. 
[27] Nelsen, R.B. 2006. An Introduction to Copulas. 2nd Edition. In: Springer Series in Statistics. New York: Springer.

[28] Prabhu N.U. 1998. Stochastic Storage Processes: Queues, Insurance Risk, Dams, and Data Communication. New York: Springer.

[29] Psarrakos, G. and Politis, K. 2012. The covariance between the surplus prior to and at ruin in the classical risk model. ASTIN Bulletin 42(2): 631-653.

[30] Resnick, S.I. 1992. Adventures in Stochastic Processes. Boston: Birkhauser.

[31] Sparre Andersen, E. 1957. On the collective theory of risk in the case of contagion between claims. In: Proceedings of the Transactions of the XVth International Congress on Actuaries vol. II, New York, pp. 219-229.

[32] Willmot, G.E. 2007. On the discounted penalty function in the renewal risk model with general interclaim times. Insurance: Mathematics and Economics 41(1): 17-31.

[33] Willmot, G.E. And Woo, J.-K. 2012. On the analysis of a general class of dependent risk processes. Insurance: Mathematics and Economics 51(1): 134-141.

[34] Woo, J.-K. 2010. Some remarks on delayed renewal risk models. ASTIN Bulletin 40(1): 199-219.

[35] Woo, J.-K. 2012. A generalized penalty function for a class of discrete renewal processes. Scandinavian Actuarial Journal 2012(2): 130-152.

[36] Woo, J.-K. And Cheung, E.C.K. 2013. A note on discounted compound renewal sums under dependency. Insurance: Mathematics and Economics 52(2): 170-179.

[37] Zhang, Z., Yang, H. And Yang, H. 2012. On a Sparre Andersen risk model with time-dependent claim sizes and jump-diffusion perturbation. Methodology and Computing in Applied Probability 14(4): 973-995. 
$\operatorname{Cov}\left(\tau, Z_{0.01}(\tau) \mid u\right)$

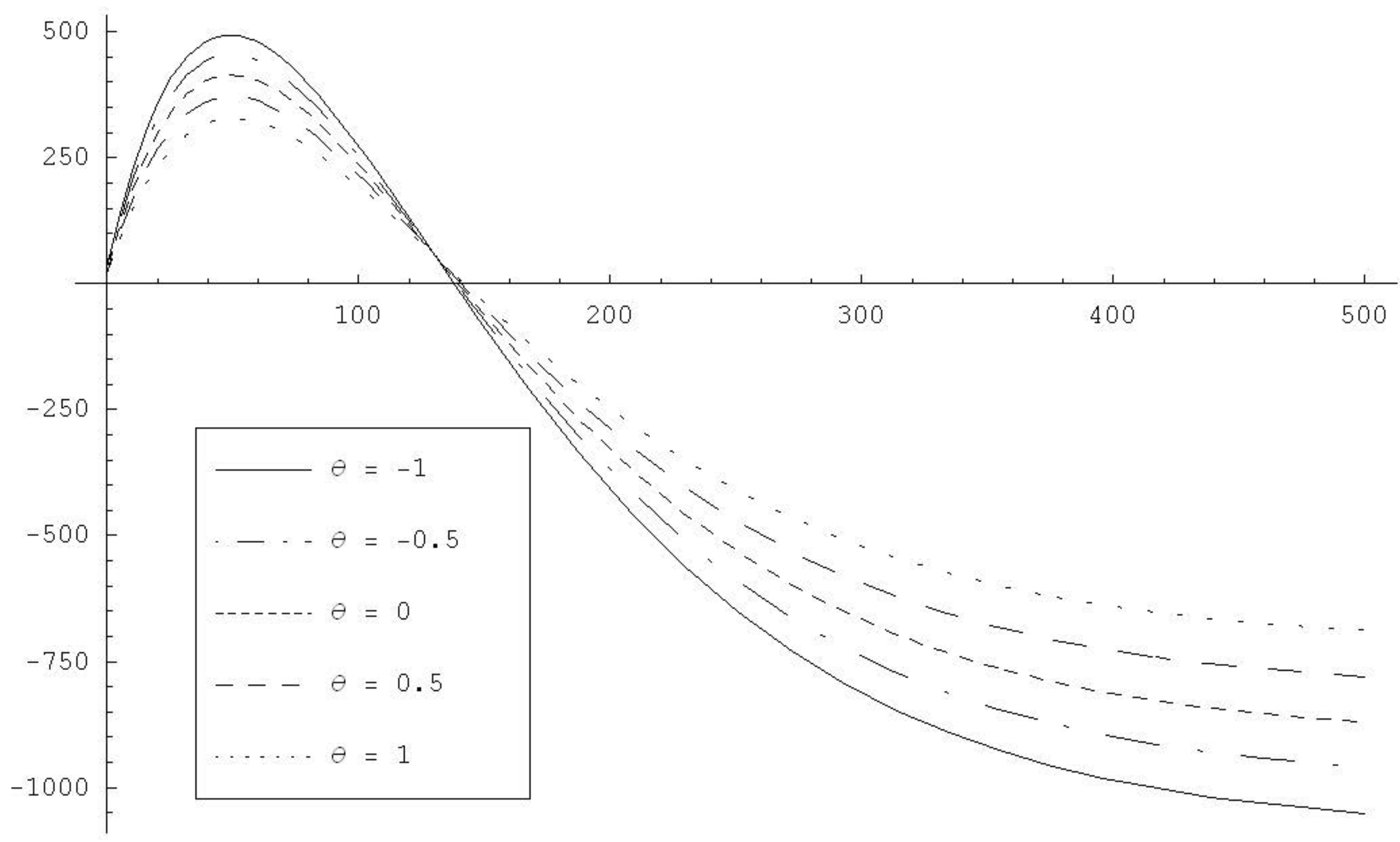


$\operatorname{Cov}\left(\tau, z_{0.05}(\tau) \mid u\right)$

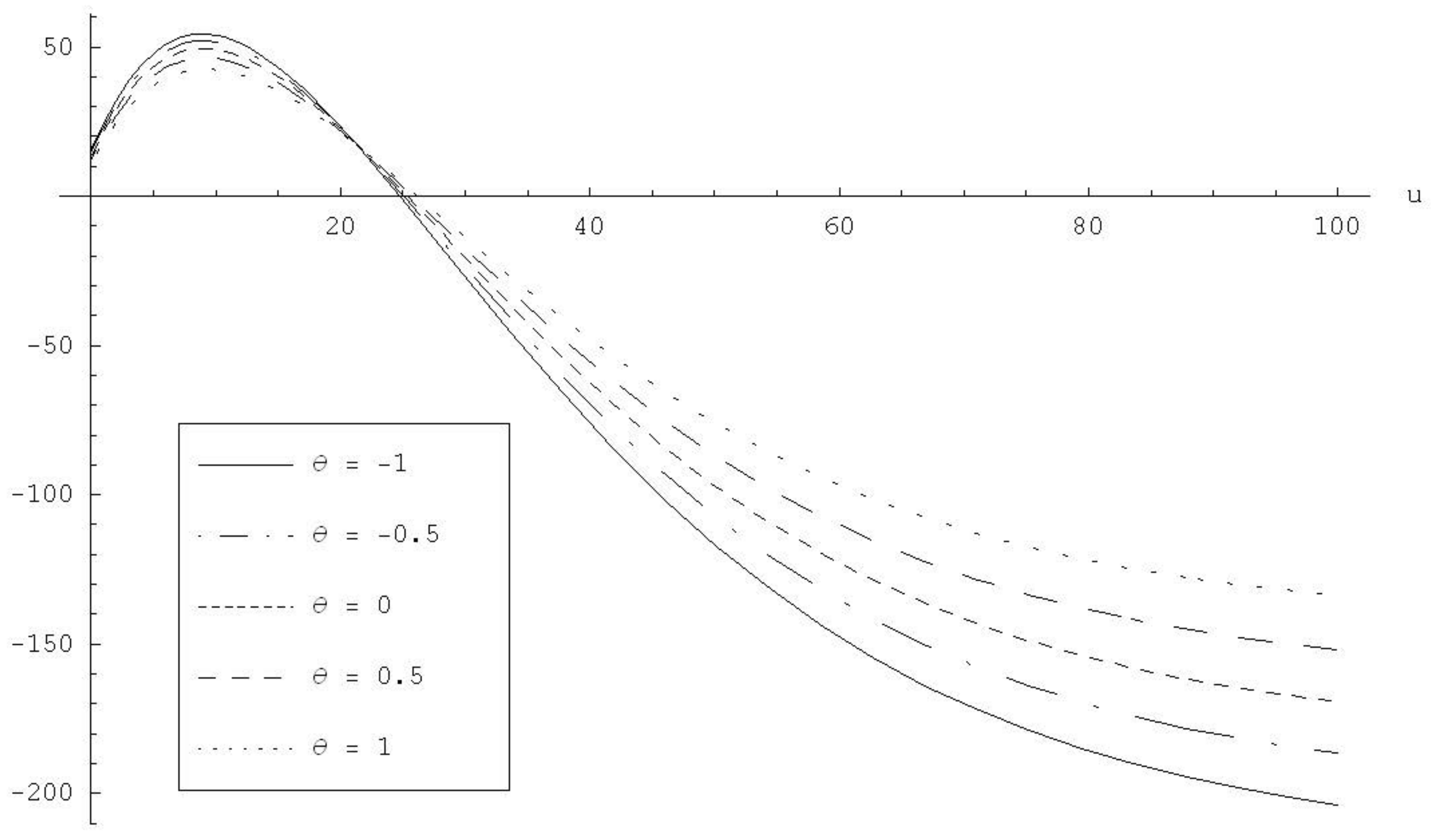


$\operatorname{Mean}\left(Z_{0.01}(\tau) \mid u\right)$

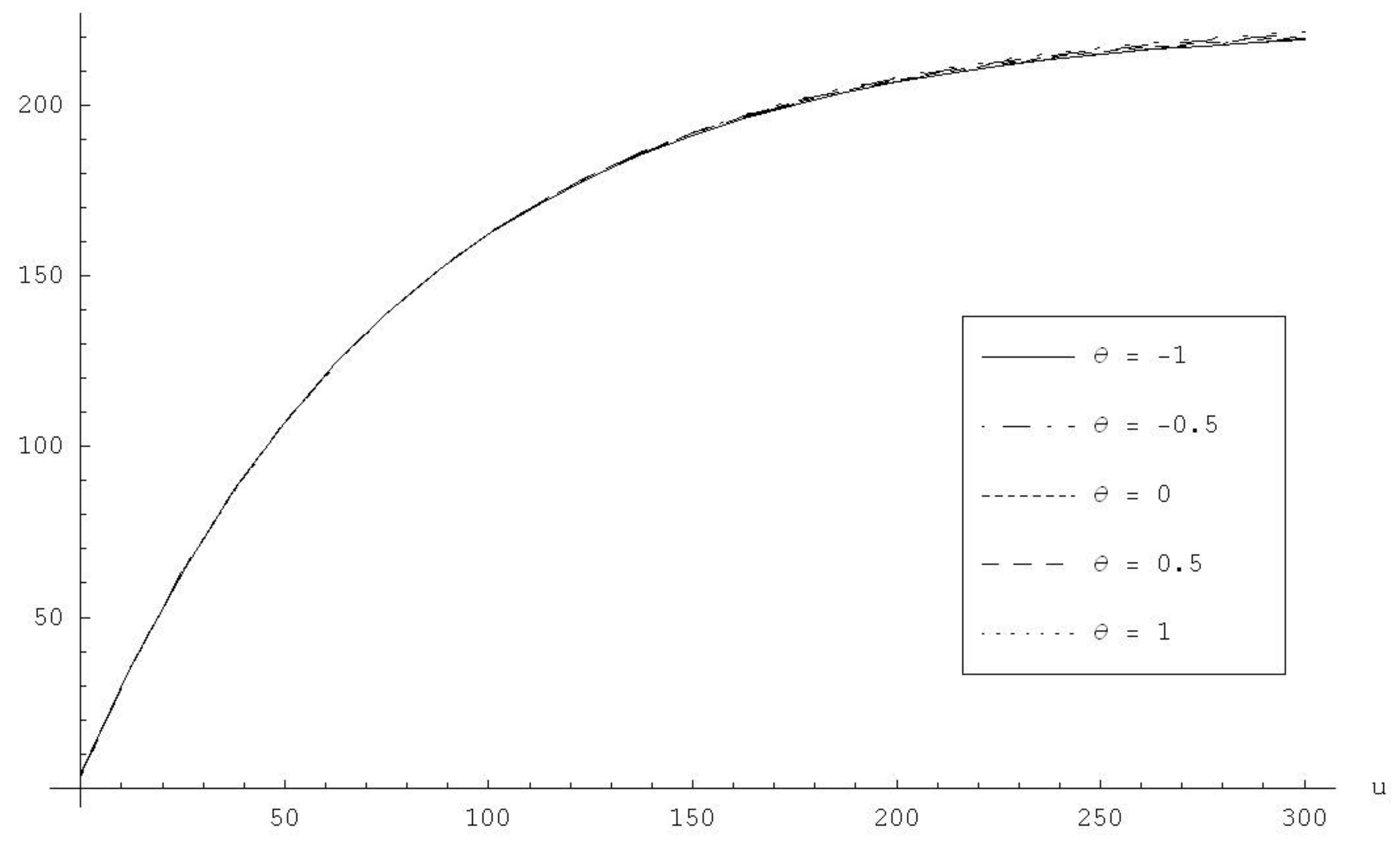


$\operatorname{Mean}\left(Z_{0.05}(\tau) \mid u\right)$

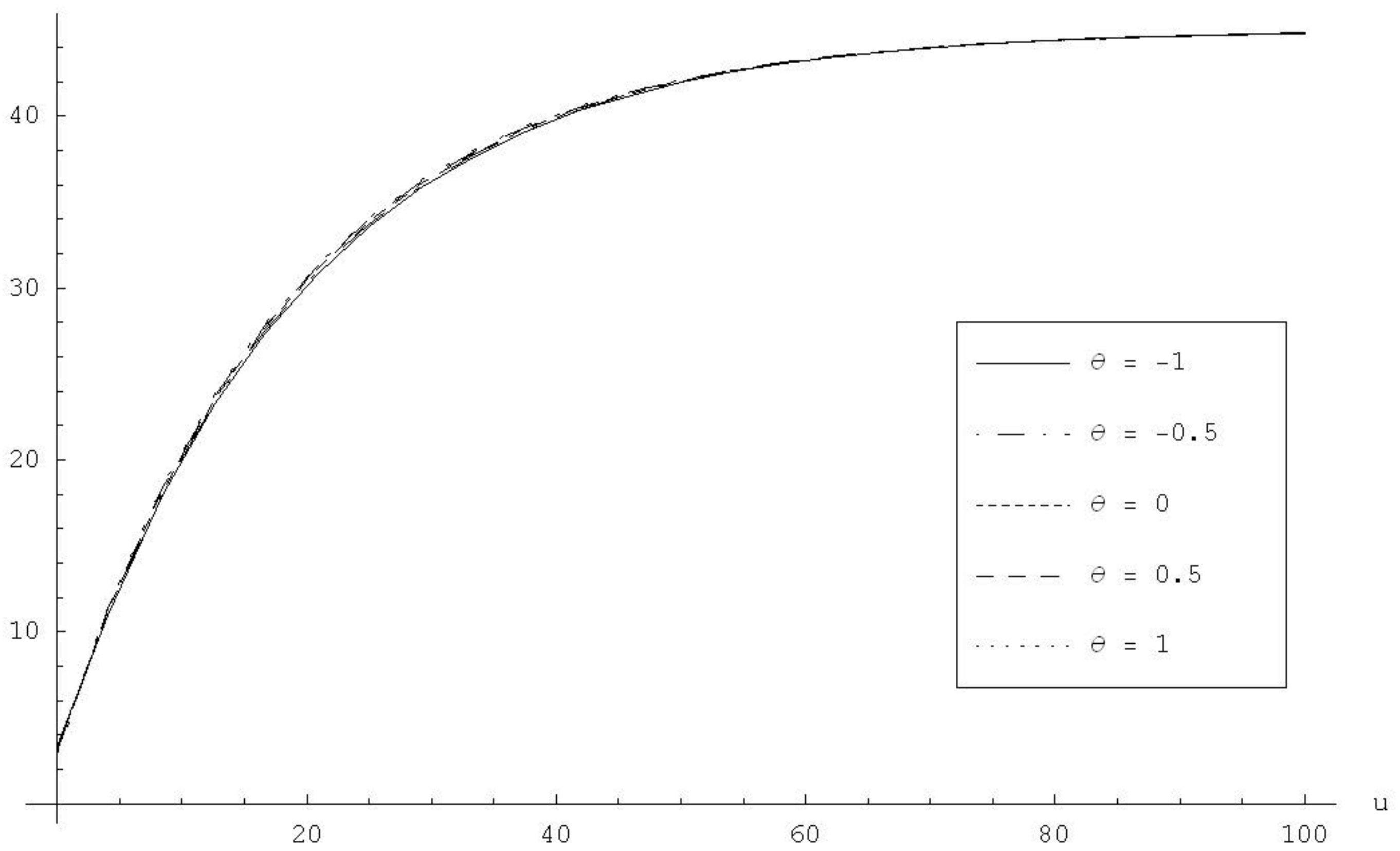


$\operatorname{Var}\left(\mathrm{Z}_{0.01}(\tau) \mid \mathrm{u}\right)$

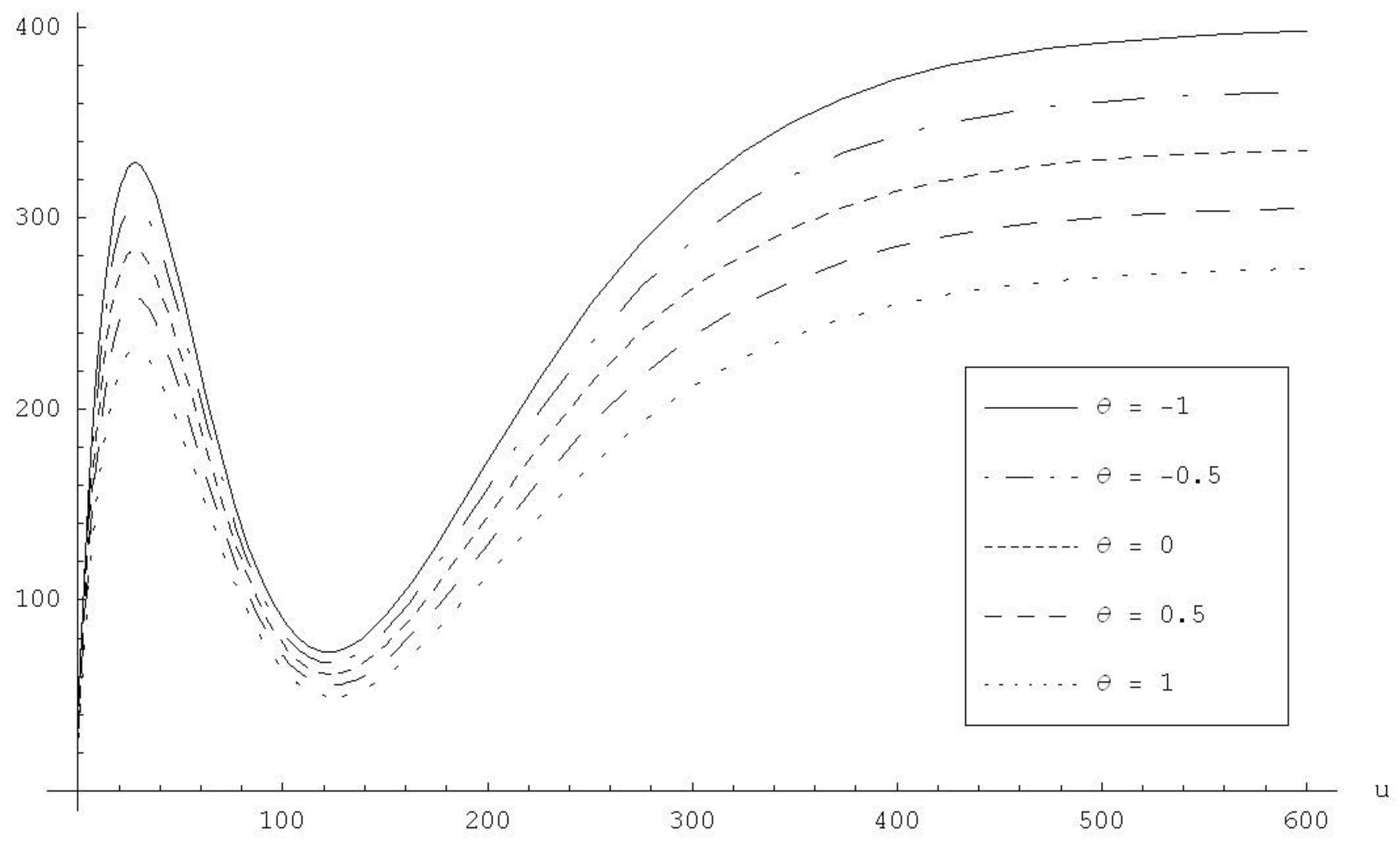


$\operatorname{Var}\left(Z_{0.05}(\tau) \mid u\right)$

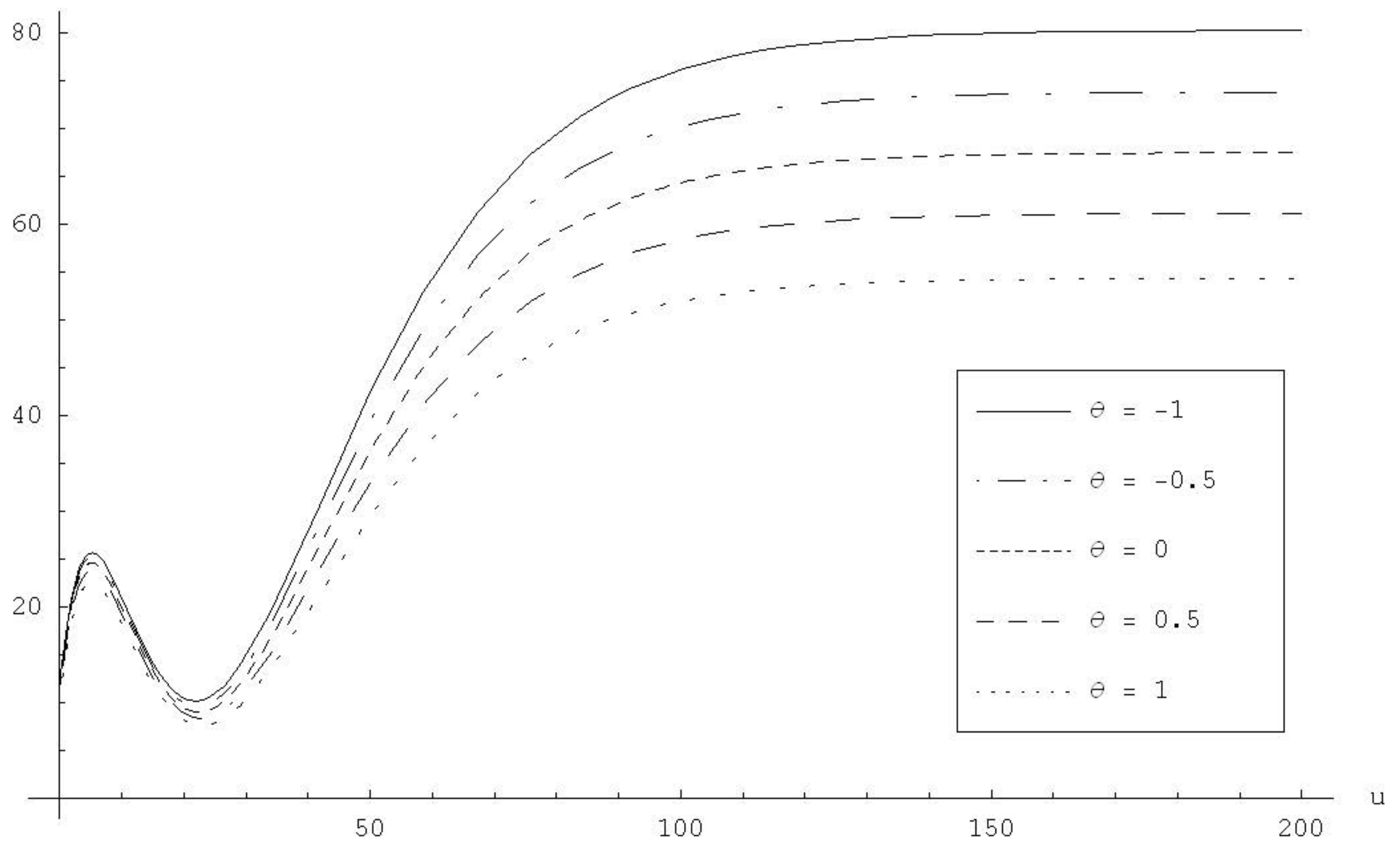


$\mathrm{CM}_{3}\left(Z_{0.01}(\tau) \mid u\right)$

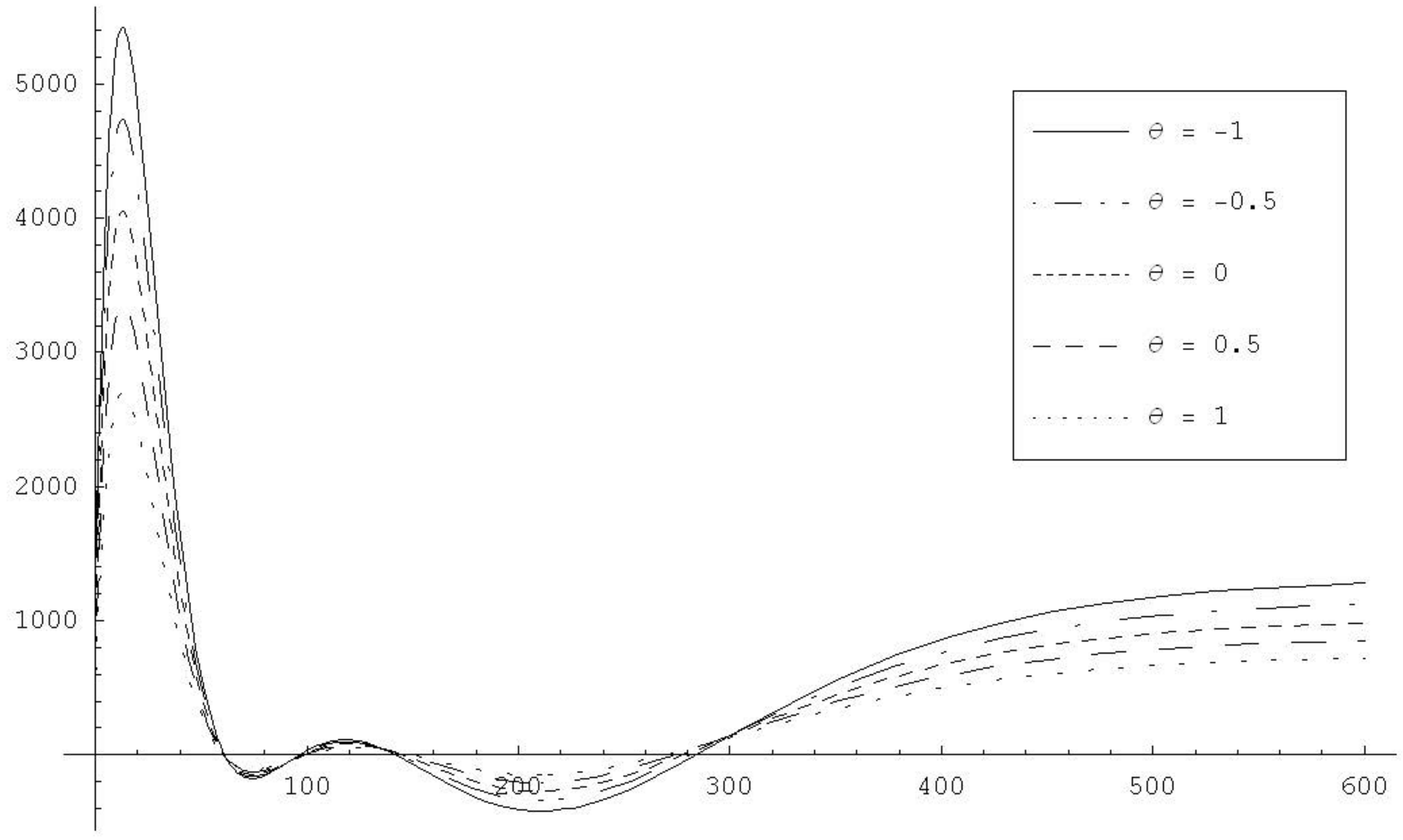


$\mathrm{CM}_{3}\left(\mathrm{Z}_{0.05}(\tau) \mid \mathrm{u}\right)$

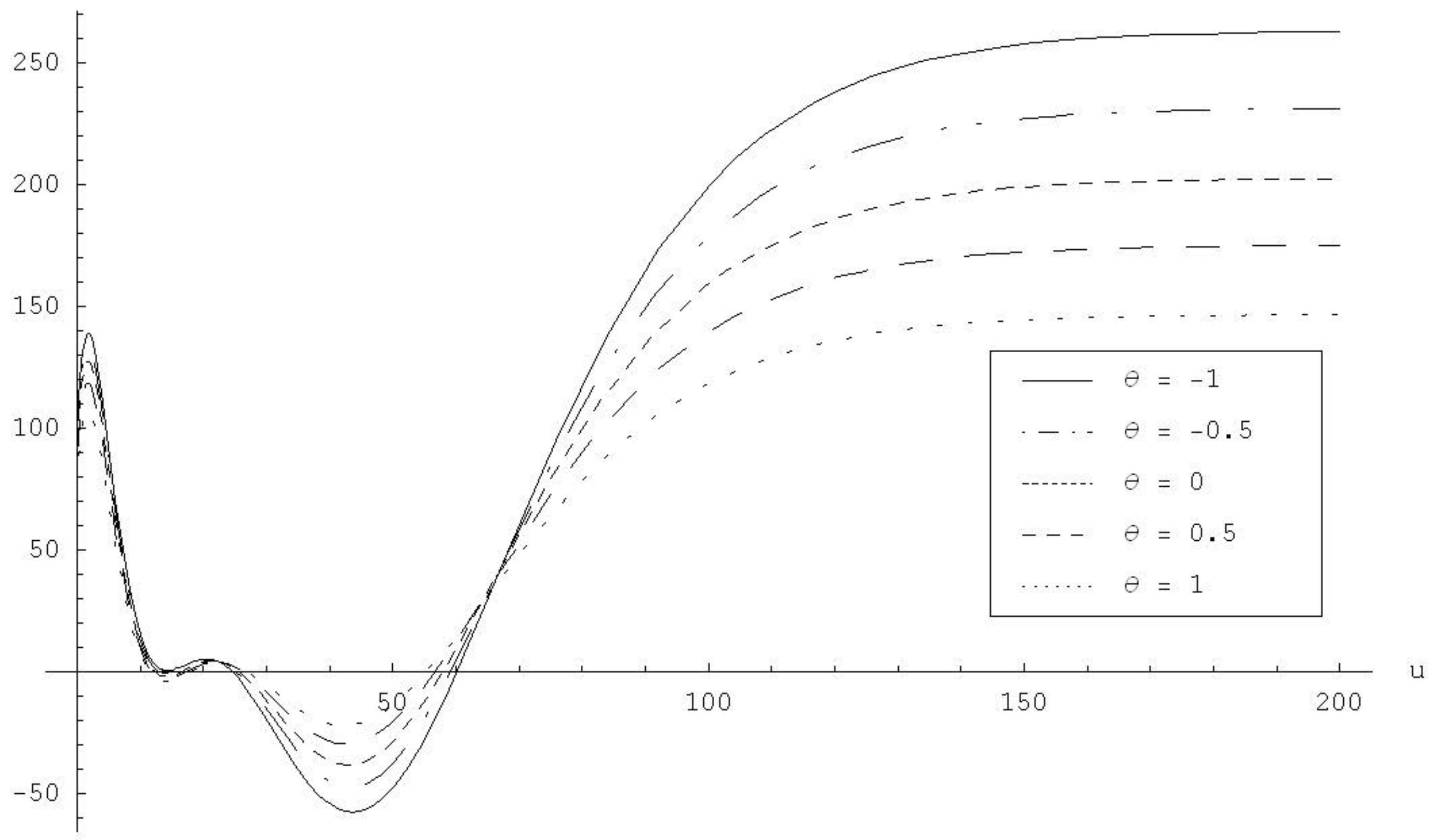

